\title{
An Experimental Study on Lateral Acceleration of Cars in Different Environments in Sichuan, Southwest China
}

\author{
Jin $\mathrm{Xu},{ }^{1}$ Kui Yang, ${ }^{2}$ YiMing Shao, ${ }^{1}$ and GongYuan $\mathrm{Lu}^{2}$ \\ ${ }^{1}$ College of Traffic and Transportation, Chongqing Jiaotong University, Chongqing 610031, China \\ ${ }^{2}$ School of Transportation and Logistics, Southwest Jiaotong University, Chengdu 610031, China \\ Correspondence should be addressed to GongYuan Lu; 834703417@qq.com
}

Received 16 July 2014; Accepted 25 September 2014

Academic Editor: Yongjun Shen

Copyright (C) 2015 Jin Xu et al. This is an open access article distributed under the Creative Commons Attribution License, which permits unrestricted use, distribution, and reproduction in any medium, provided the original work is properly cited.

\begin{abstract}
In view of the tremendous improvements in the dynamic performance of automobiles and changes in the composition of drivers over the past several decades, it is valuable to examine the distribution of automobile's lateral acceleration in new environments. The lateral accelerations, speeds, and trajectory curvatures of a passenger car on twelve highways with different design speeds and topographies were obtained. By synchronizing, matching, and counting, the distribution of the lateral acceleration was obtained, and the level of driving comfort was estimated. The 10th, 15th, 50th, 85th, and 90th percentile values of the lateral acceleration on three road types (six-lane road, four-lane road, and two-lane road) were found. Combined with scatter diagrams of the lateral acceleration over the curvature, the negative correlation between the lateral acceleration and the curvature was analyzed and interpreted. In addition, regression models of the lateral acceleration with the curvature for the three kinds of roads were established. Based on scatter diagrams of the lateral acceleration over the speed, regression models of the average $a_{y}$ depending on the speed and the 85th percentile $a_{y}$ depending on the speed of passenger cars and buses were established. The research provides calculation parameters and a control basis for operating speed prediction, pavement material design, and maximum and minimum limit value determination for the superelevation ratios and curve radii.
\end{abstract}

\section{Introduction}

In highway engineering, surveying the lateral accelerations of vehicles and determining the change rules for their distributions and influencing factors require significant work. It is valuable to study the lateral acceleration of a moving vehicle. First, the lateral acceleration can be converted into the lateral force coefficient of a highway, which is a key factor for lateral stability and driving safety. Some suggestions can then be given for the design of highway pavement, to improve its performance for a sufficient lateral friction coefficient. Second, by comparing with the permissible values of acceleration that ensure human comfort, the measured acceleration can be used to evaluate the rationality of a highway's superelevation. This is helpful to determine the applicable range of superelevation ratios. Third, the lateral acceleration is directly related to the lateral stability of a vehicle. The driving behavior on a curve, such as the selection of trajectory and speed is largely affected by the magnitude of lateral force acting on the driver, therefore a thoriugh investigation on lateral acceleration can present basic parameters for predicting the trajectory and speed on a complicated highway. Fourth, as the most direct and objective measurement of lateral comfort, the lateral acceleration is related to the horizontal alignment of a highway. The model of the relationship between the lateral acceleration and the alignment of a highway can be used to predict the lateral comfort of a route scheme in the design stage. This is helpful to improve the geometric design for better performance.

In recent years, lateral acceleration has been examined from multiple perspectives all over the world. The lateral accelerations of a passenger car on an interchange ramp, a ring road of a city expressway, and a rugged mountain road were measured by Hugemann and Nicke from Germany [1]. The 10th, 50th, and 90th percentile values of the lateral acceleration were obtained to present the distribution characteristic. In addition, a model of the relationship between the lateral acceleration and the radius of a curve was obtained. 
However, no universal conclusions could be obtained for the design practice because of the insufficient sample size. The lateral acceleration of a passenger car was also studied by Tokunaga et al., from the highway department of the Hokkaido Research Center in Japan. They studied a mountain highway with a length of $5 \mathrm{~km}$, including 16 curves with radii ranging from $50 \mathrm{~m}$ to $530 \mathrm{~m}$. The distribution of the acceleration on each curve was obtained by repeated experiments, but their focus was on the influence of the vehicle navigation system on the driver's behavior [2].

Carlson and Mason Jr. used a ball bank indicator to investigate the lateral accelerations and roll angles of typical passenger cars. Their results showed that ball bank indicator readings and lateral acceleration values were highly correlated, and the influence of body-roll on ball bank indicator readings appeared to be negligible when using typical passenger cars to determine safe speeds on horizontal curves [3]. Bonneson conducted a field survey at horizontal curves and collected data on the driving speed, headway, curve radius, superelevation rate, and grade. One of his conclusions was that the superelevation distribution method demonstrated in "A policy on Geometric Design of Highway and Streets" did not promote design consistency because of the uncomfortable lateral acceleration suffered by drivers [4]. Prior to 2004, the design values for the side friction factor used to determine a curve radius and superelevation rate were based on studies conducted in the 1930s and 1940s. However, the drivers population and passenger car designs have changed significantly over the last 50 years. Therefore, Tan conducted an experiment wherein male and female subjects of various ages were driven around three horizontal curves at a test track and asked to indicate when they felt uncomfortable. The $10 \%, 50 \%$, and $90 \%$ uncomfortable lateral acceleration values were then determined [5].

He et al., an engineer from China, measured the sideway force coefficient (SFC) using a sideway force coefficient routine investigation machine (SCRIM) for different speeds on a particular highway. The statistical regression model between SFC and the speed was obtained in this way [6]. Pan et al. conducted an experiment using eleven horizontal curves on a mountain road [7]. The collected data demonstrated how the increase in the heart rate and the maximal blood pressure changed over a lateral acceleration. A statistical model of the driving workload was obtained in this experiment, with SFC as the argument. In 2002, a monographic study on SFC was promoted by the Research Institute of Highway in China. The SFCs of three typical vehicles-a passenger cars, buses, and trucks-were obtained. The SFC curve over the speed was obtained and adopted in the following design specifications for highways, which provides some references for the value range of the superelevation ratio [8].

However, the traffic composition has changed greatly in recent years, with a significant reduction in the proportion of trucks and buses. Because of the economic development in the past decade, the proportion of passenger cars has increased greatly, and the driver composition has become more diverse. The ratio of private cars has changed from being negligible to being the biggest. The age and driving year distributions have become more scattered than in the past.
In addition, great improvements have been made in vehicle performance, including the acceleration performance, braking performance, and handling dynamics, which make higher speeds for passenger cars attainable. The driving performance of a passenger car is different from that of a truck or bus, which means its lateral acceleration characteristics are different too. Thus, it is necessary to survey and analyze the lateral acceleration for better highway designs adapted to vehicle performance and driving behavior.

It took two years for us to collect lateral acceleration data from vehicles in Sichuan, southwest China. The technical parameters of the measured highways were different, with different terrain environments, types, design speeds, and technical grades. This paper is organized as follows: the design of the data collection and extraction method is discussed in Section 2; the distribution characteristics of the lateral acceleration are presented in Section 3; and Section 4 shows how the lateral acceleration changed with the radius of a vehicle's trajectory and with its speed. The way the relationship was influenced by the terrain environment, technical grade, and number of lanes on a roadway was also analyzed in the process.

\section{Data Collection and Extraction}

Generally, sufficient on-site data can be obtained from a sample survey to reach significant, rational, and universal experimental conclusions. The lateral acceleration, trajectory, speed, and other parameters were surveyed as initial data in the experiment, as shown in Figure 1. They were recorded by specific vehicle-loaded equipment that moved continuously along with its host.

2.1. Tested Highway. Twelve typical roadways were surveyed in Sichuan, southwest China. The trajectory, speed, and acceleration were obtained to determine the driving properties. The roadways had different technical grades, terrain environments, and number of lanes; the key technical characteristics are shown in Table 1.

2.2. Type of Vehicle. Passenger cars, buses, and trucks were leading in the traffic composition of a previous survey, with passenger cars having the biggest share. In Sichuan, passenger cars constitute $60 \%-80 \%$ of the vehicles on expressways and highways with two lanes and $85 \%-90 \%$ during festivals and holidays. Trucks and buses follow, with buses slightly ahead of trucks on roads to best known scenic spots. The lateral acceleration data and results for passenger cars are presented in this paper, while those for buses and trucks will be presented in the next two papers. The types of passenger cars investigated in this study included sedans, hatchbacks, sport utility vehicles, multipurpose vehicles, and minibuses (with less than 9 seats).

2.3. Measuring Method. The vehicle performance data were measured using an MTi microinertial attitude heading reference system. There were nine kinds of data collected: the three-axis acceleration, pitch angular, pitch angular velocity, 


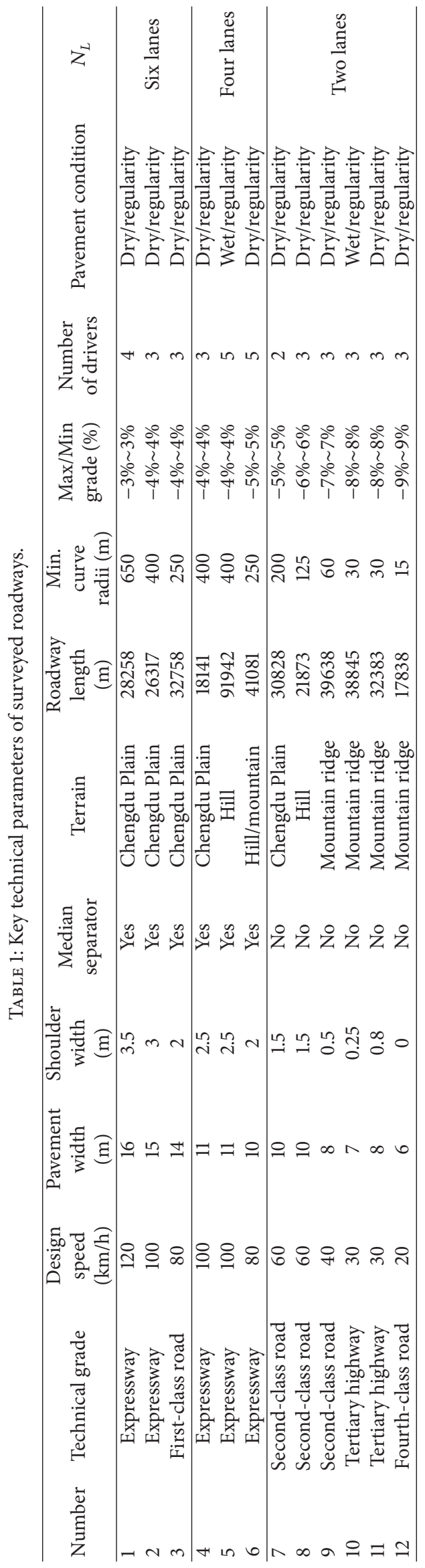



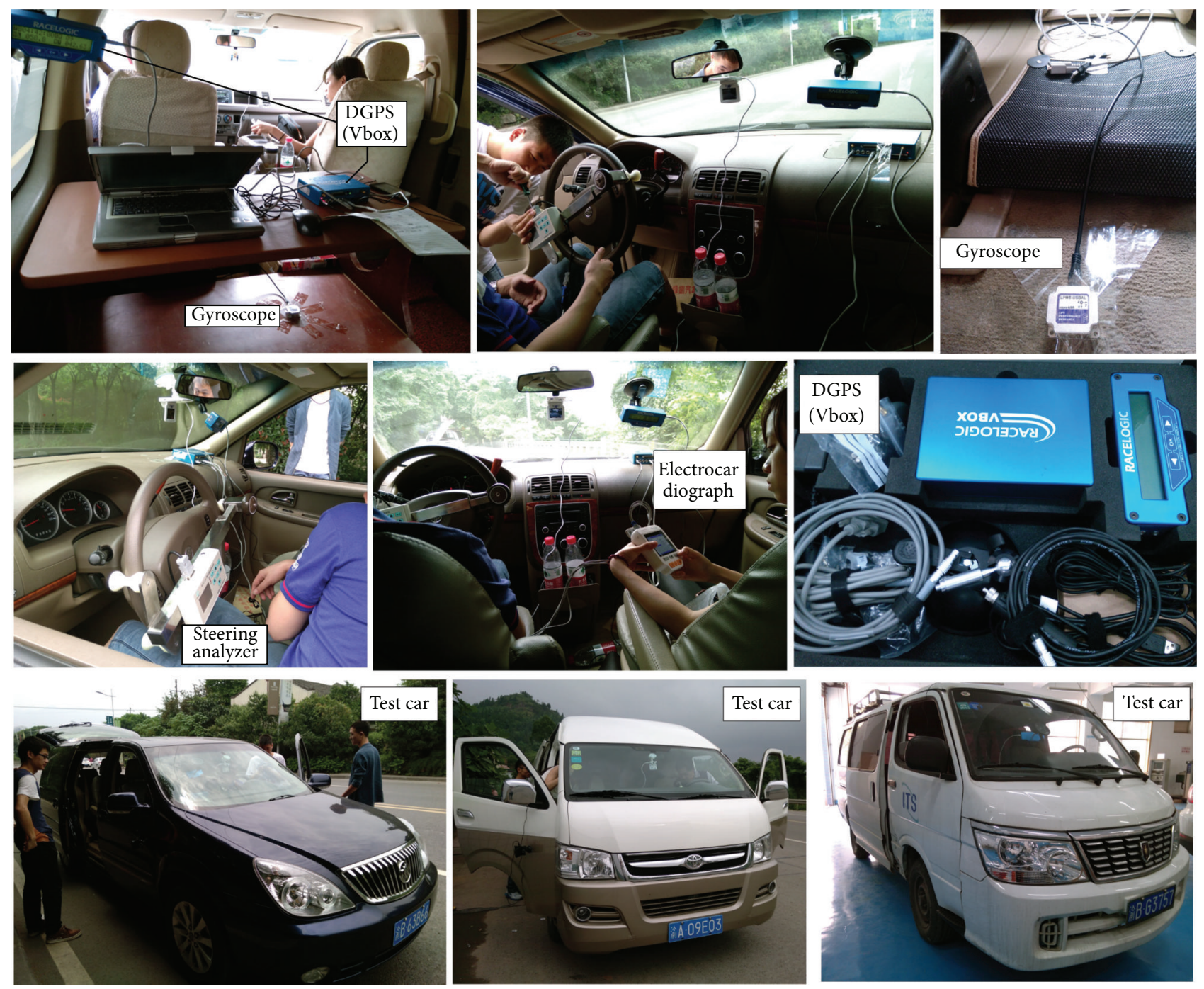

FIGURE 1: Collecting operating parameters of passenger cars driving on different highways.

TABLE 2: Basic information for surveyed drivers.

\begin{tabular}{lccccccc}
\hline Type & Number & Male & Formal & Age & Average age & Driving years & Average driving years \\
\hline Professional & 8 & 6 & 2 & $26-49$ & 35 & $3-21$ & 13 \\
Unlicensed & 15 & 13 & 2 & $25-46$ & 35 & $2-15$ & 7 \\
Private & 17 & 9 & 8 & $26-50$ & 33 & $1-12$ & 3.5 \\
\hline
\end{tabular}

yaw, yaw velocity roll angle, and roll angle velocity. In this study, the lateral acceleration is analyzed. A sensor was installed on the floor of the vehicle, and about 30 min was spent in calibrating and commissioning the instruments to reduce the error. The driving speed was recorded using a noncontact speedometer that was installed on the headstock or tailstock. The trajectory was recorded using a highprecision dual-frequency differential global positioning system (DGPS). The coordinate data of the trajectory were converted to the curvature at each location.

2.4. Drivers. The subjects were all volunteers and included three types of passenger car drivers: professional, unlicensed, and private. The term professional referred to professional cab drivers working for a specific company. The unlicensed drivers made a living by transporting passengers with their private automobiles, without an operating license. Unlicensed drivers constitute a large proportion of the total, especially in some rural areas of China. However, private drivers constitute a greater proportion and have poorer driving skills and less experience.

Because of the influence of the driver on the driving behavior, different types of drivers were recruited to determine the difference between the driving behaviors. The basic information for the surveyed drivers is listed in Table 2. 
2.5. Data Extraction. The time series were included in the data from the microinertial attitude heading reference system, noncontact speedometer, and DGPS, all on a millisecond scale. A notebook computer, connected to a data acquisition card with three channels, was used to synchronously record the data from the three different pieces of equipment. This made it convenient to synchronize the data collected. The extraction and matching method is illustrated in Figure 2, where the data are from a section of a mountain highway with a length of $7500 \mathrm{~m}$. The trajectory from the DGPS, the lateral acceleration, the curvature from the coordinate data, and the continuous speed from the noncontact speedometer are shown in the first to the fourth graphs, respectively.

Generally, the lateral acceleration along a curve changes over time in the driving process, first rising and then declining, in which the peak corresponds to the center of the curve. The worst stability corresponds to the peak of the lateral acceleration, which is our focus. The pickup algorithm was designed to determine the peak point of the lateral acceleration. Using the initial data, the peak lateral acceleration and its corresponding time and mileage were obtained. The curvature and speed were determined based on the time series and mileage. Then, the data were stored in the format $\left(i, a_{y i}, k_{i}, v_{i}\right)$, in which $a_{y i}, k_{i}$, and $v_{i}$ are the lateral acceleration, curvature, and speed, respectively. Figure 2 shows that the peak of the lateral acceleration almost corresponds to the peak of the curvature, but there is a difference between the speed and the lateral acceleration. It was not difficult to draw scatter diagrams of the lateral acceleration over the curvature and speed for further statistical analyses.

\section{Distribution of Lateral Acceleration}

The lateral accelerations of the vehicles were tested on 12 highways are presented in Figure 3, and it is convenient to evaluate the safety and comfort by the distribution of the lateral acceleration.

Figures 3(a)-3(c) show the distribution of the lateral accelerations for three highways with six lanes. With three lanes in the same driving direction, it is easy for a driver to overtake a slower passenger car by changing lanes and reaching his expected speed. The lateral acceleration distributions are very similar to each other, which is a result of the almost identical driving environments in the Chengdu Plain, and have good alignments. For example, the 85th percentile values are all about $1.0 \mathrm{~m} / \mathrm{s}^{2}$, with the maximum values below $3.5 \mathrm{~m} / \mathrm{s}^{2}$. According to the previous conclusions, the level of comfort corresponds to a particular threshold value, with the lateral acceleration as the evaluation index for comfort [9-11]. The threshold value of comfort is $1.8 \mathrm{~m} / \mathrm{s}^{2}$, with medium comfort and discomfort levels of $3.6 \mathrm{~m} / \mathrm{s}^{2}$ and $5 \mathrm{~m} / \mathrm{s}^{2}$, respectively [12]. Therefore, a six-lane highway on a plain had at least a medium comfort level, and this comfort level accounted for more than $90 \%$ of the data.

Figures 3(d)-3(f) show the distributions of the lateral accelerations for the three highways with four lanes, with

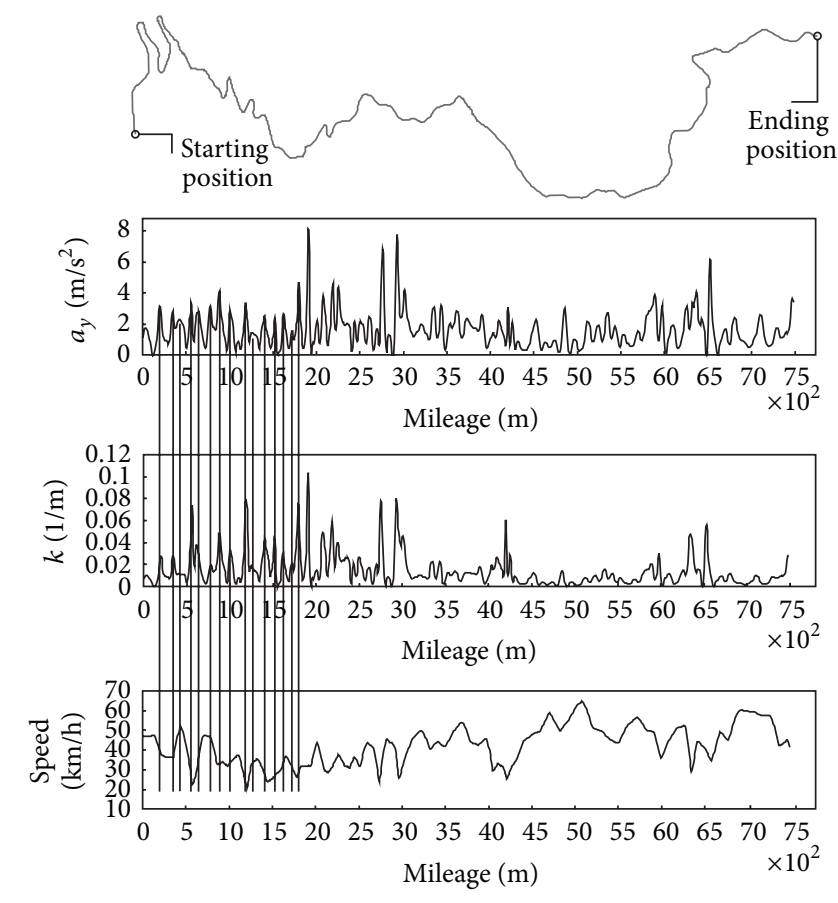

FIGURE 2: Illustration of data extraction and matching.

plain, hill, and mountain as the driving environments, respectively. The lateral accelerations with higher values accounted for the greatest percentage, with environments from plain to mountain. In the plain, the lateral accelerations were all smaller than $3.0 \mathrm{~m} / \mathrm{s}^{2}$, and most were smaller than the threshold value of comfort. For a highway in the hill area, there were few points with a lateral acceleration greater than $3.6 \mathrm{~m} / \mathrm{s}^{2}$, and the points with lateral accelerations smaller than $1.8 \mathrm{~m} / \mathrm{s}^{2}$ accounted for about $85 \%$. However, the medium comfort level had a much higher proportion, and this comfort level accounted for less than $85 \%$ in the mountain environment.

Figures $3(\mathrm{~g})-3(\mathrm{l})$ show the distributions of the lateral accelerations of the six highways with two lanes. The lateral accelerations of the highways with two lanes were much greater than those of the highways with four or six lanes. The distribution of the lateral acceleration was greatly affected by the terrain environment because small-radius curves are commonly used on mountain highways with two lanes to reduce the excavation and embankment volume.

It is comfortable to drive on highway number 7 on a plain and highway number 8 on a hill. The comfort level for highway number 9 in a mountain area was considerable beyond the medium comfort value and a few points beyond the discomfort level. In contrast, the medium comfort level accounted for about $15 \%$ on highways number 10 , number 11 , and number 12, with a much greater proportion a few points beyond the discomfort threshold value. A reduction in comfort was evident on a mountain highway, especially at a speed of less than $30 \mathrm{~km} / \mathrm{h}$ (the last three highways). At least $15 \%$ drove with a medium comfort level, and in certain individual sections, the comfort level was unacceptable. 


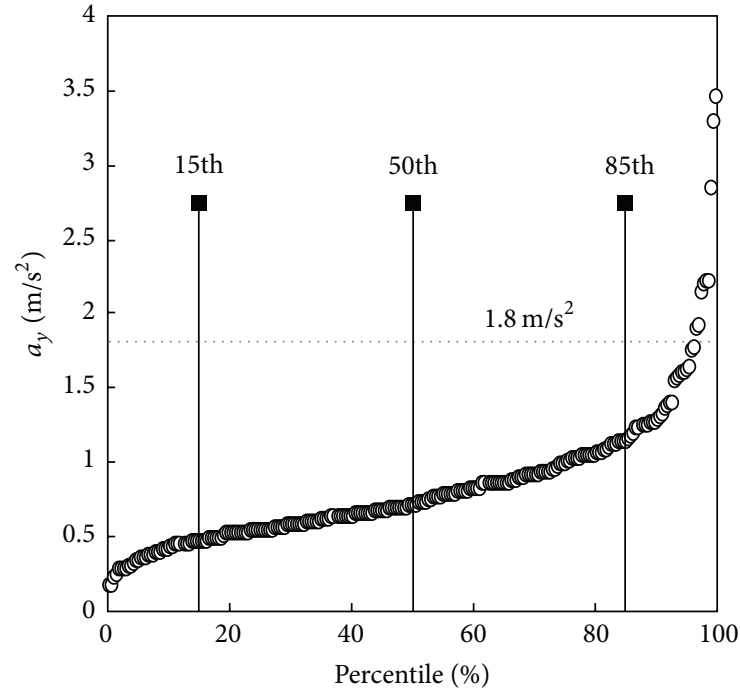

(a) Highway number 1

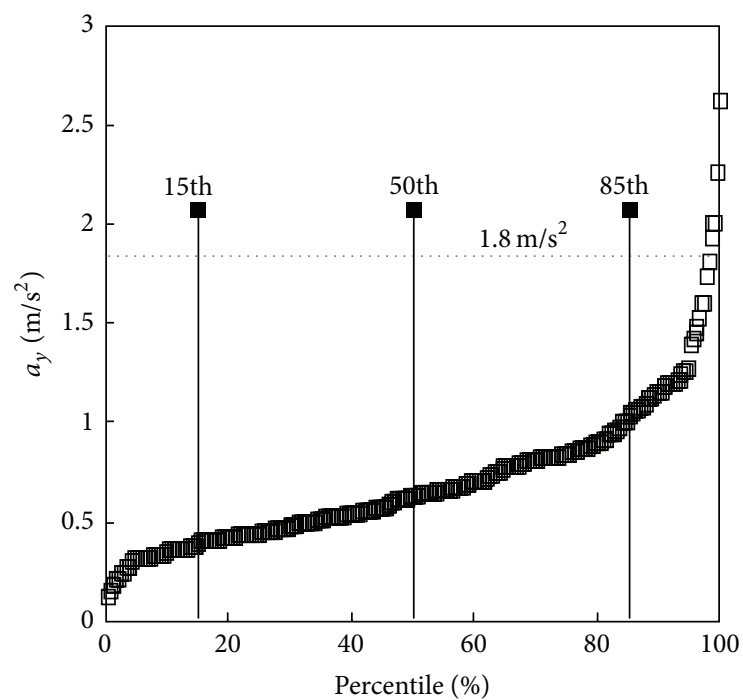

(c) Highway number 3

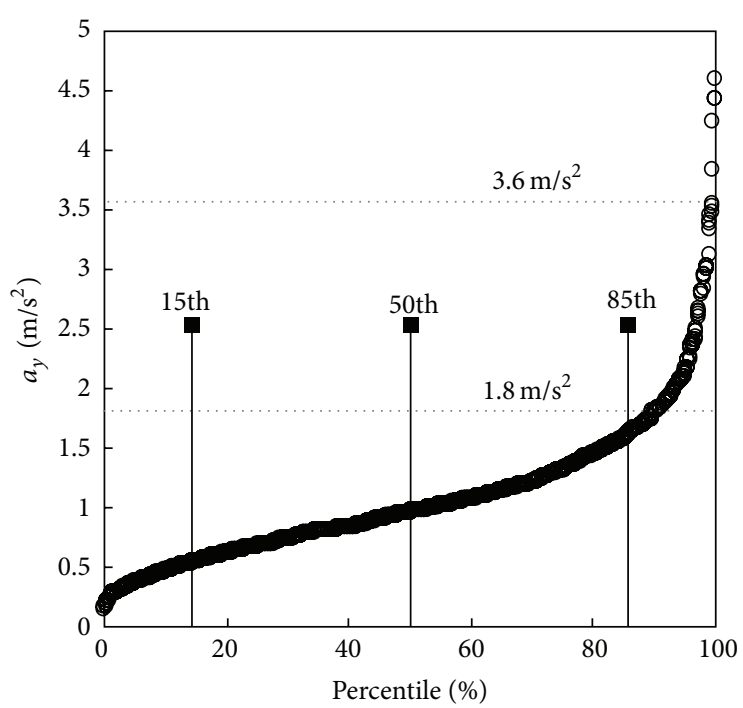

(e) Highway number 5

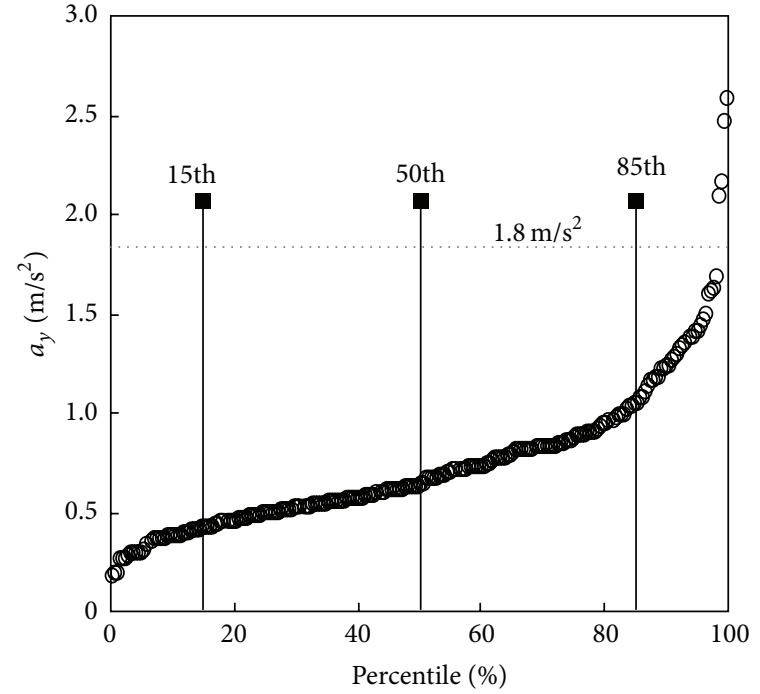

(b) Highway number 2

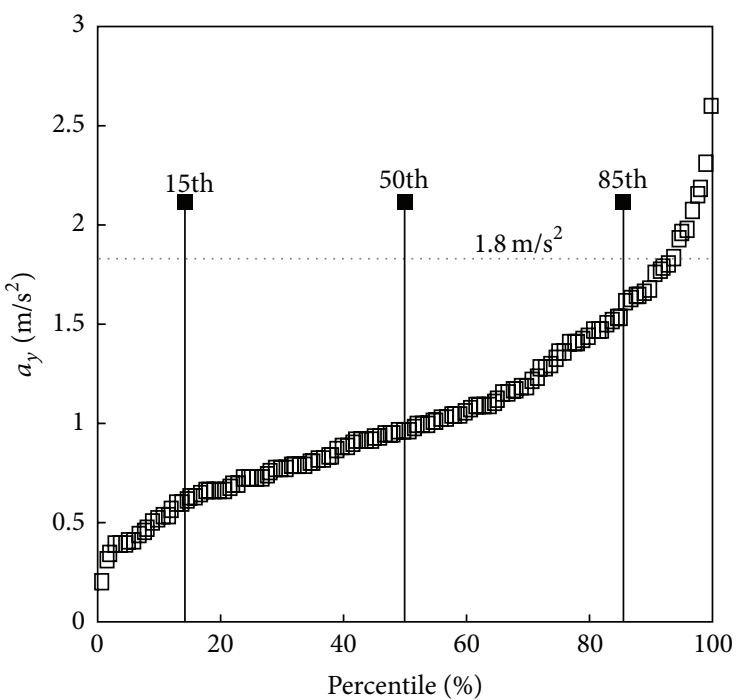

(d) Highway number 4

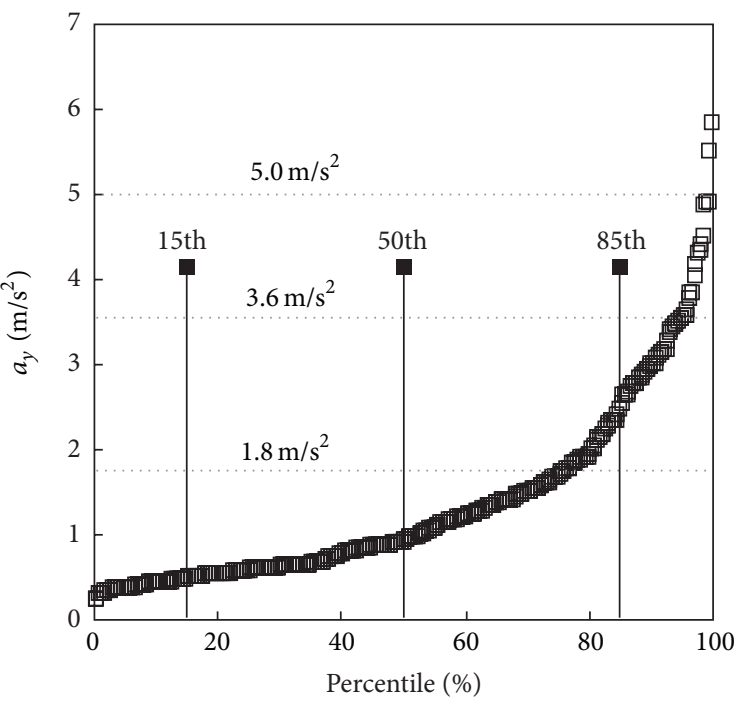

(f) Highway number 6

FIgURE 3: Continued. 


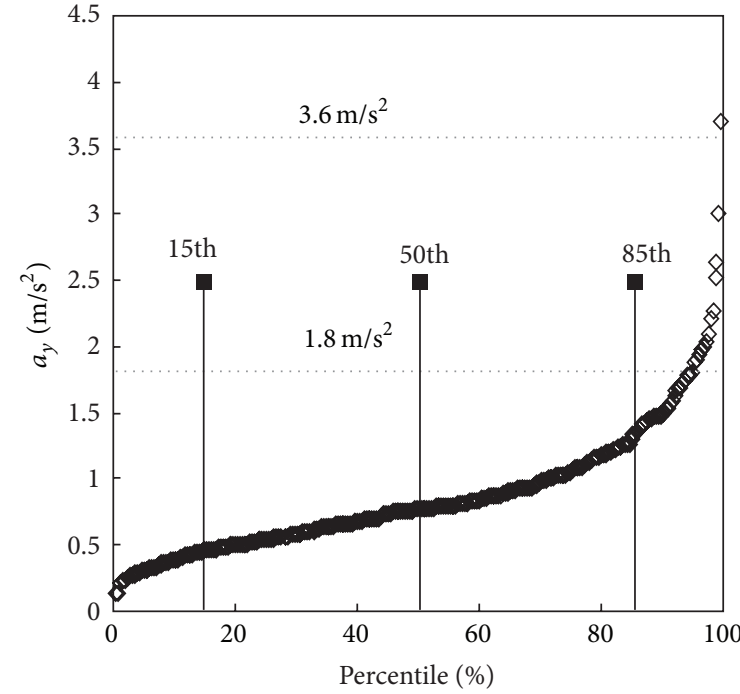

(g) Highway number 7

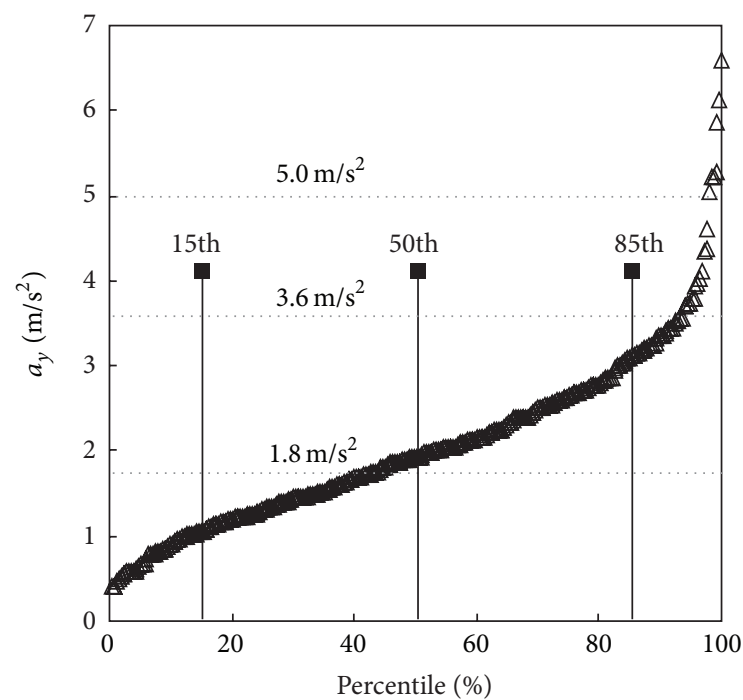

(i) Highway number 9

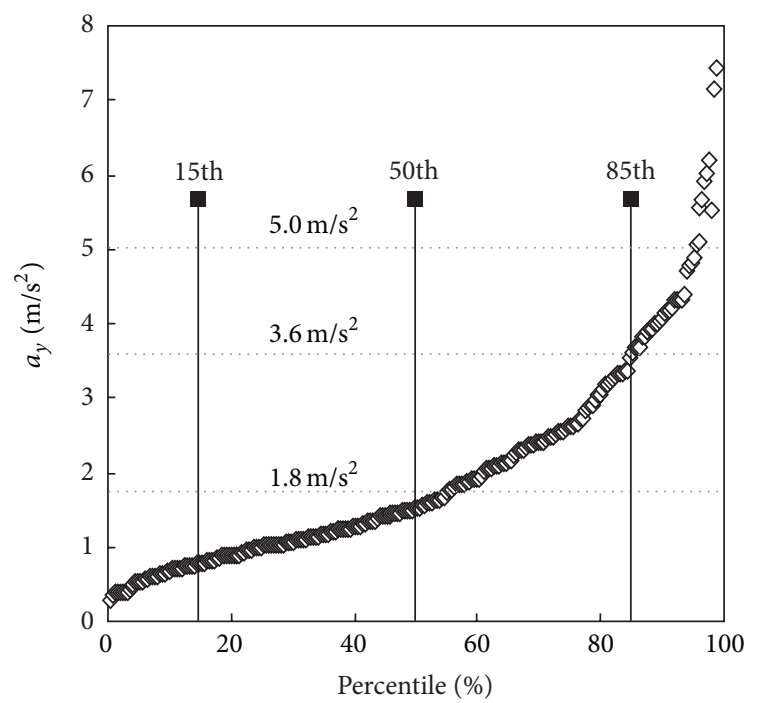

(k) Highway number 11

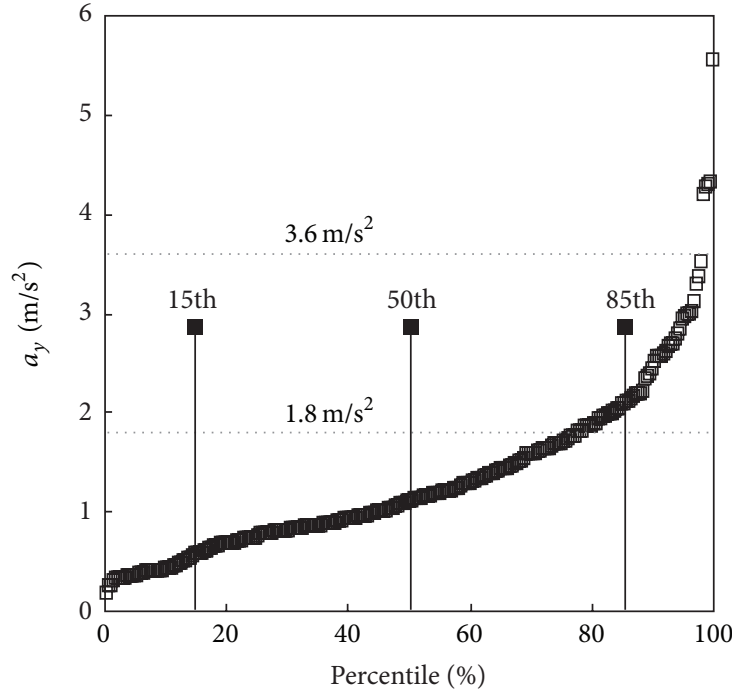

(h) Highway number 8

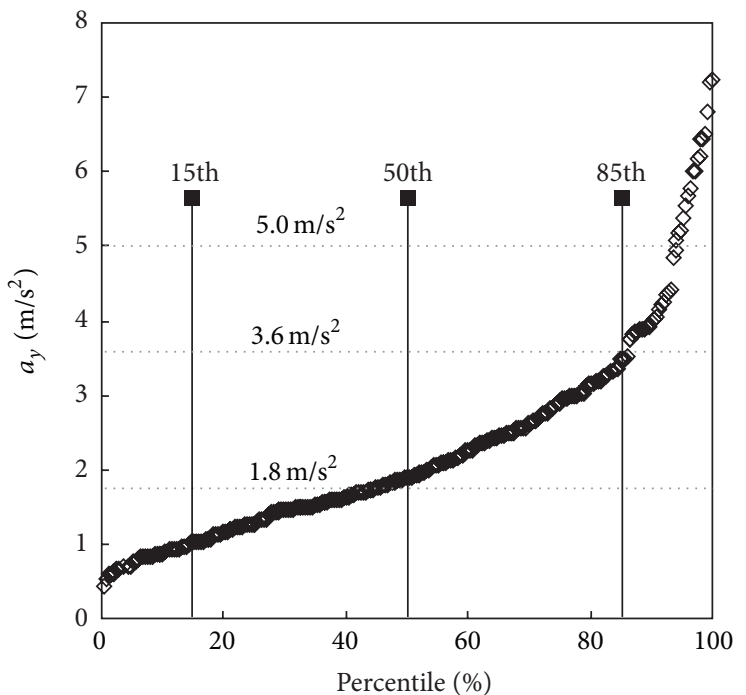

(j) Highway number 10

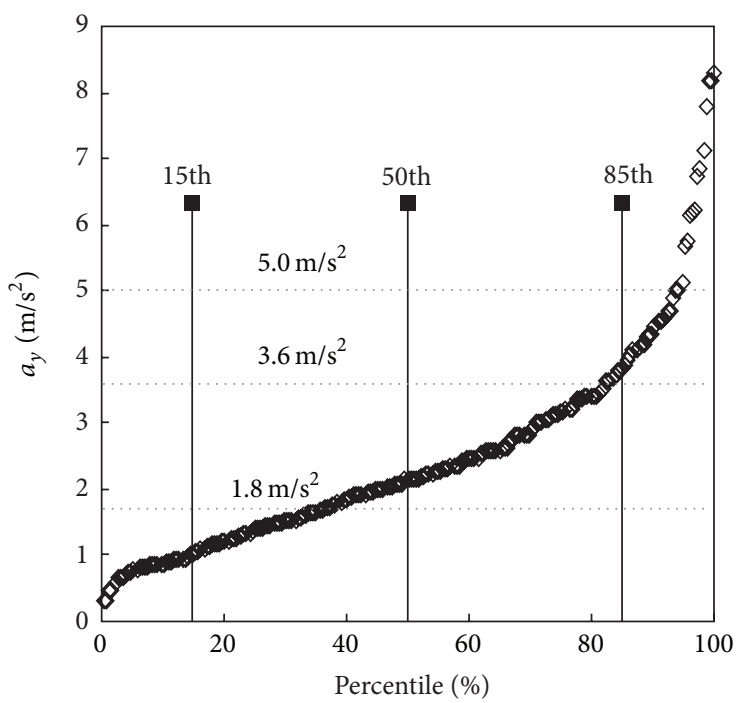

(l) Highway number 12

FIGURE 3: Distribution of lateral accelerations. 


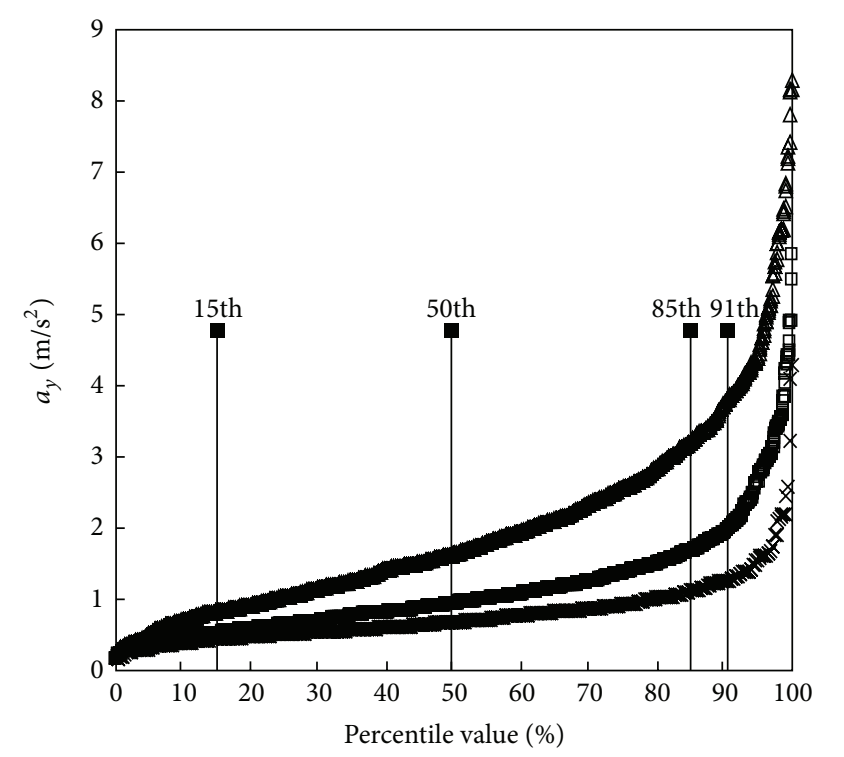

$\triangle$ Two lanes

$\square$ Four lanes

$\times$ Six lanes

FIGURE 4: Distributions of lateral accelerations for highways with different lanes.

TABLE 3: Percentile values of lateral acceleration.

\begin{tabular}{lccc}
\hline Percentile & \multicolumn{3}{c}{$\begin{array}{c}\text { Percentile value } \\
\text { Four lanes }\end{array}$} \\
\hline 10th & 0.38614 & 0.450358 & 0.702805 \\
15th & 0.445396 & 0.525151 & 0.830999 \\
50th & 0.683107 & 0.941924 & 1.637689 \\
85th & 1.121519 & 1.67618 & 3.201153 \\
90th & 1.256985 & 1.933957 & 3.730245 \\
\hline
\end{tabular}

The lateral accelerations of 12 highways were aggregated by the number of lanes, as shown in Figure 4 . This shows a smooth S-shaped curve for highways with the same number of lanes, with an inflection point at about the 90th percentile. It should be noted that inflection point did not occur at the 85 th percentile, as in the previous research, and it is valuable to evaluate the usage of the 85 th percentile value in road geometry design.

The percentile values of the lateral accelerations on the three different highways are presented in Table 3 (10th, 15th, 50 th, 85th, and 90th). The percentile values were all below $2 \mathrm{~m} / \mathrm{s}^{2}$, except for the 85th and 90th on a highway with two lanes. It will be helpful for the percentile value to be adopted for the geometric design of highways.

\section{Effect of Curve Radius on Lateral Acceleration}

A lateral acceleration accompanies driving around a curve. Because of the freedom for the selection of trajectory and speed in highway travelling, the lateral acceleration is influenced by the driving behavior. It is valuable to study the influence of the radius on the lateral acceleration.

The measured lateral acceleration of a passenger car is shown in Figure 5, in which the figure on the left is a scatter diagram of the lateral acceleration over the curvature. The lateral acceleration increases with the curvature. The lateral acceleration is concentrated with a small curvature and becomes scattered with an increase in the curvature. For a designer, the radius is much more intuitive than the curvature, and the left scatter diagram can be transformed into the right diagram, the scatter diagram of the lateral acceleration over the radius, with remarkable regularity.

The scatter diagram of the lateral acceleration over the radius of all twelve highways is presented in Figure 6. Despite the number of lanes, there is a strong nonlinear negative correlation between the lateral acceleration and the radius. It is well understood that a higher speed is accompanied by a greater radius, and accidents are much more serious than those at lower speeds. Thus, it is better for a driver to drive with a small lateral acceleration and greater safety margin for lateral stability.

The lateral acceleration of a highway with six lanes is relatively concentrated and becomes scattered with a reduction in the number of lanes. The great similarity of the environment and geometric alignment of highways with six lanes leads to a higher consistency for the driving behavior. However, the geometric alignment and width are different on highways with two lanes, especially in mountain areas. The great difference between different highways leads to different driving behaviors, in addition to scattered lateral accelerations.

The lateral accelerations $a_{y i}$ were aggregated by the type of highway according to the number of lanes (six, four, or two). The results of the normal distribution tests for the three sets of data using the SPSS 19.0 software are presented in Figure 7. The data show an abnormal distribution because there appeared to be significant differences between the histogram and normal distribution curve. Because lateral acceleration data are not subject to a normal distribution, a parametric test such a $T$-Test cannot be used to test for the significance. For this reason, the Kruskal Wallis Test, which is a nonparametric test method, was selected to test the significance among the three sets of $a_{y i}$ data. The significance values of each group of data are listed in Table 4, where an "Asymptotic Significance" value equal to zero indicates a significant difference among the $a_{y i}$ data for the highways with different number of lanes.

Scatter plots of $a_{y i}$ versus the curve radii of the three types of highways are shown in Figure 8. The distribution is much more concentrated and regular, which makes it convenient for us to set up a relationship model between the lateral acceleration and the radius. The average model $a_{y}$ ave and the extremum model $a_{y \text { max }}$ were set up using statistical regression and envelop methods, respectively. The regression parameters were obtained by minimizing the fitting error and extreme value from the enveloped line of the scatter diagram. The average value represents the average level of driving behavior, and the extreme value represents the upper bound. 

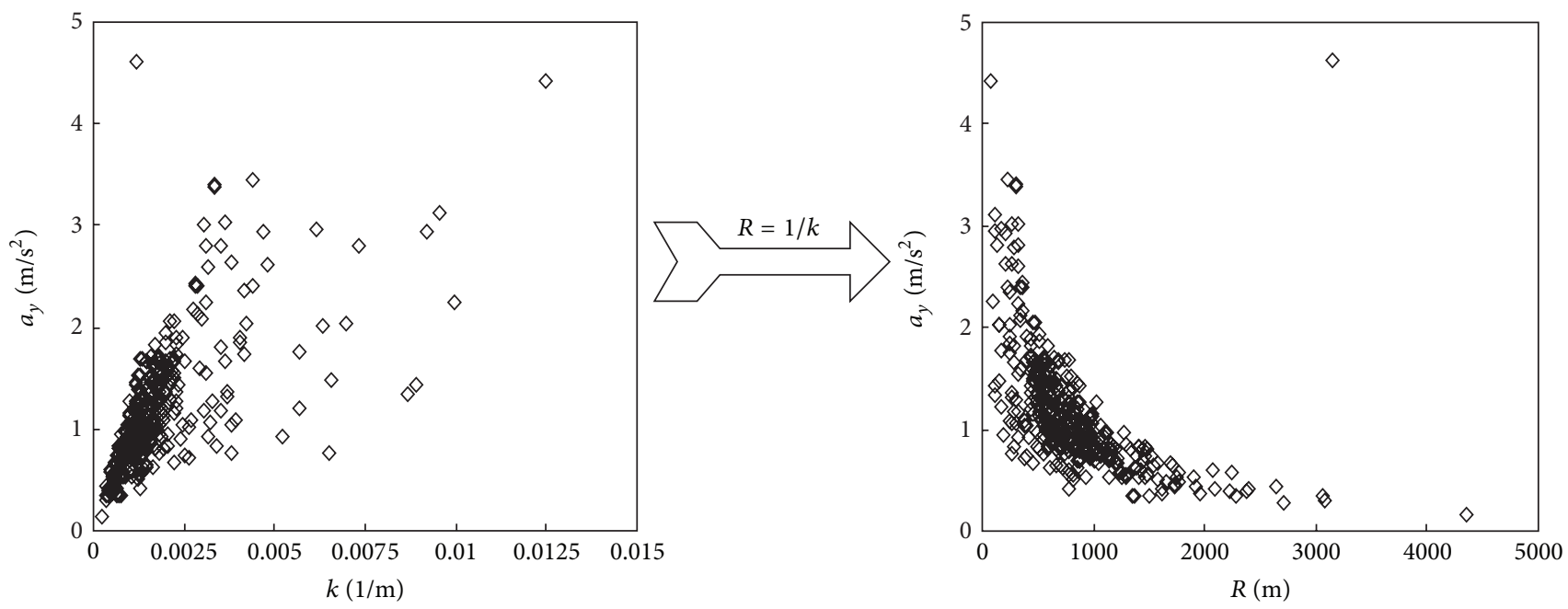

FIgURE 5: Relationships between lateral acceleration and curvature/radius.

TABLE 4: Results of Kruskal Wallis Test of significance.

\begin{tabular}{lcccc}
\hline & KW & & \multicolumn{2}{c}{ Test statistics } \\
Highway type & Sample number & Mean of KW & $a_{y}$ & 725.673 \\
\hline Six-lane highway & 472 & 921.51 & df & 2 \\
Four-lane highway & 1490 & 1384.93 & Asymptotic Significance & 0.000 \\
Two-lane highway & 1238 & 2118.82 & \\
\hline
\end{tabular}

TABLE 5: Functions of lateral acceleration over radius.

\begin{tabular}{lll}
\hline Type of highway & Function form & Value of coefficient \\
\hline Six lanes & $f(x)=a+c / x^{b}$ & $\begin{array}{l}a_{y \text { ave }}: a=0.07 ; b=0.94 ; c=550 \\
a_{y \text { max }}: a=0.07 ; b=0.91 ; c=590\end{array}$ \\
\hline Four lanes & $f(x)=a+1 /\left(x^{b}+c\right)$ & $\begin{array}{l}a_{y \text { ave }}: a=-1.8 ; b=0.0583 ; c=-1.135 \\
a_{y \text { max }}: a=-2.2 ; b=0.055 ; c=-1.17\end{array}$ \\
\hline Two lanes & $f(x)=a x^{b}+c$ & $\begin{array}{l}a_{y \text { ave }}: a=20.5 ; b=-0.46 ; c=-0.35 \\
a_{y \text { max }}: a=28.5 ; b=-0.43 ; c=-0.70\end{array}$ \\
\hline
\end{tabular}

The functions of the lateral acceleration over the radius are represented in Table 5, in which the average and extreme values can be referenced for the average and extreme values of the geometric parameter, respectively.

\section{Effect of Driving Speed on Lateral Acceleration}

The lateral acceleration depends on the driving speed in a certain curve segment. Thus, it is valuable to study the correlation between the lateral acceleration and the speed. A specific correlation model is expected to provide a reference for the design of a superelevation and speed control algorithm for an intelligent vehicle. The scatter diagrams $\left(a_{y}-V\right)$ for the three road types were obtained using the method shown in Figure 2; they are illustrated in Figure 9.
The lateral acceleration on a highway with six lanes was clustered over the speed, from $80 \mathrm{~km} / \mathrm{h}$ to $140 \mathrm{~km} / \mathrm{h}$, which indicates that the lateral acceleration was not correlated with the speed. The maximum speed on a highway with four lanes was almost the same as that on a highway with six lanes, with a much lower minimum speed. The distribution was strongly regular, and it was easy to obtain an envelope. The distribution for a highway with two lanes was much more regular, with $115 \mathrm{~km} / \mathrm{h}$ as the maximum speed.

The aggregated scatter diagram (Figure 10(a)) was obtained by gathering data from the three road types discussed above. With strong regularity, the outlines of the distribution areas were sketched using three lines. The speed section was divided into subsections with intervals of $10 \mathrm{~km} / \mathrm{h}$. 


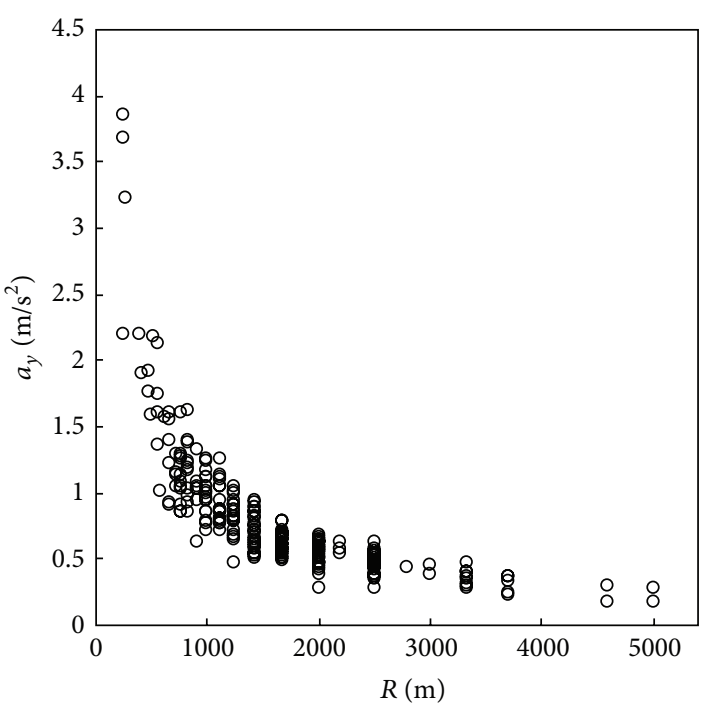

(a) Highway number 1

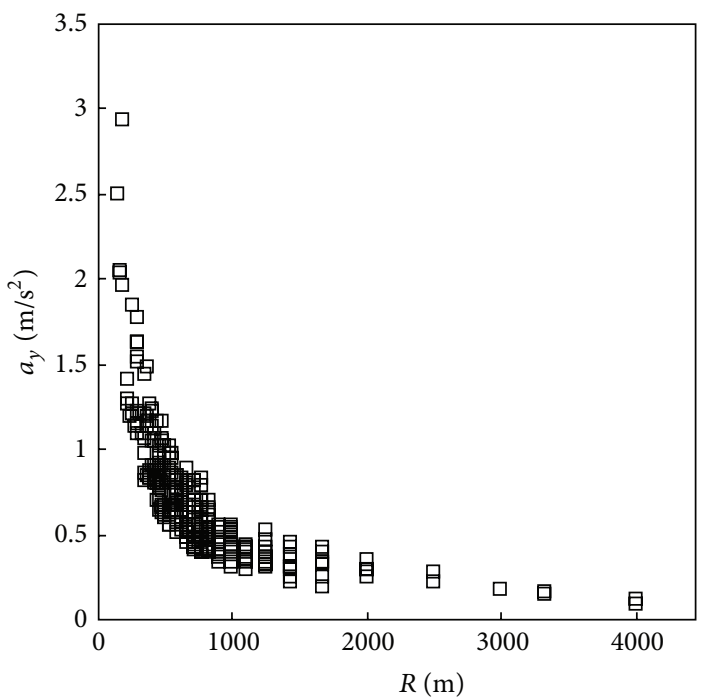

(c) Highway number 3

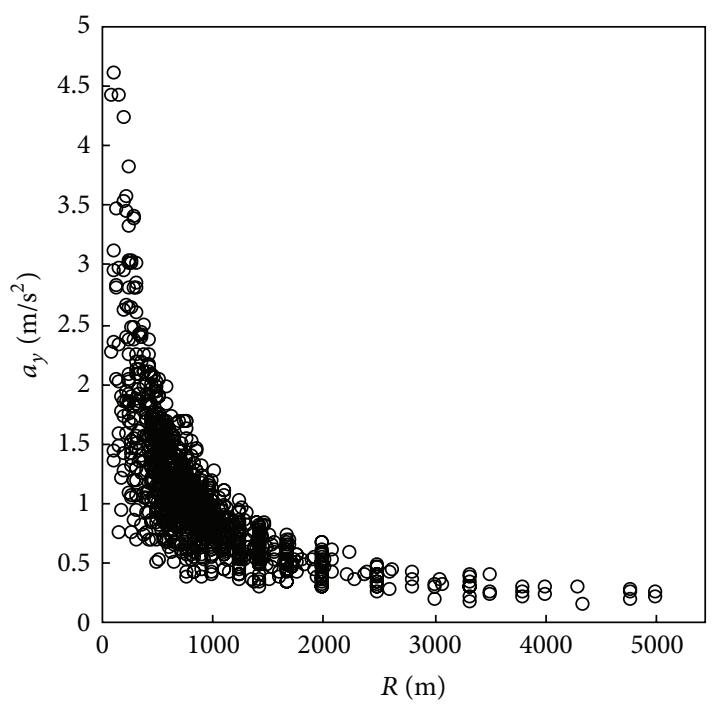

(e) Highway number 5

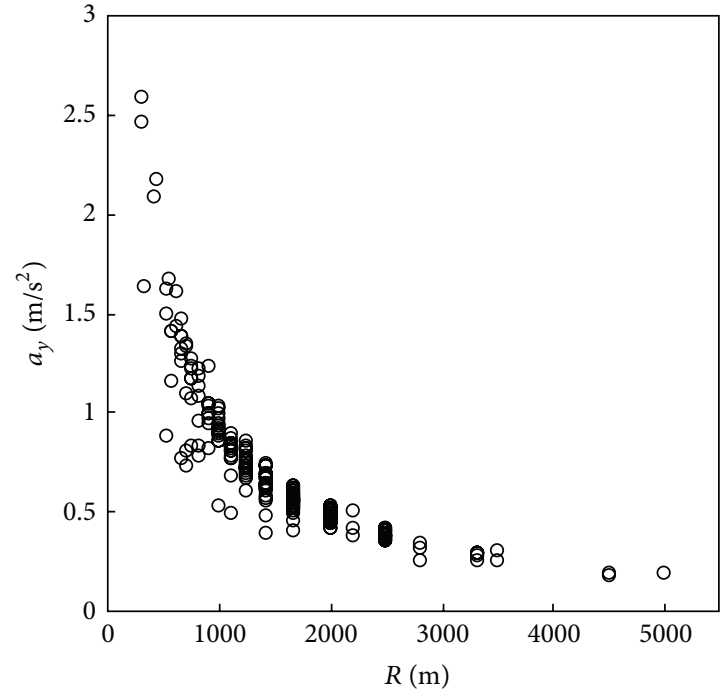

(b) Highway number 2

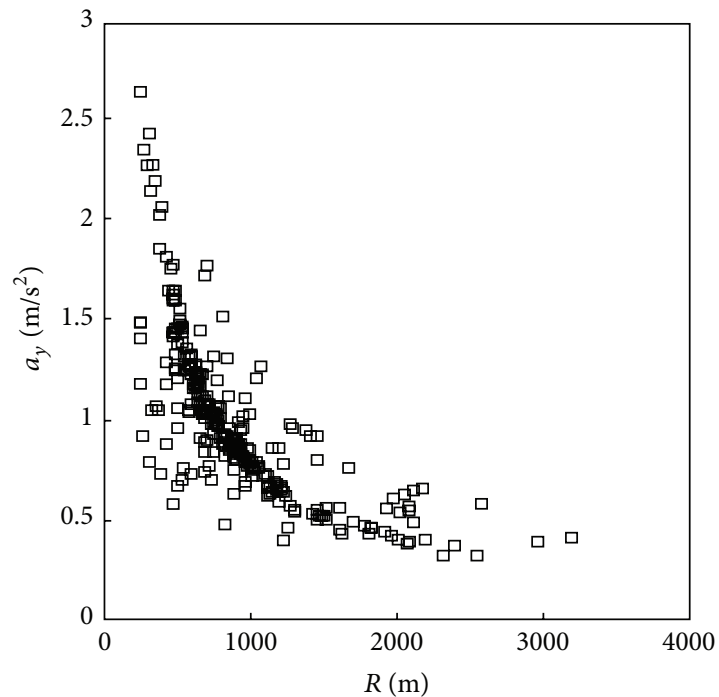

(d) Highway number 4

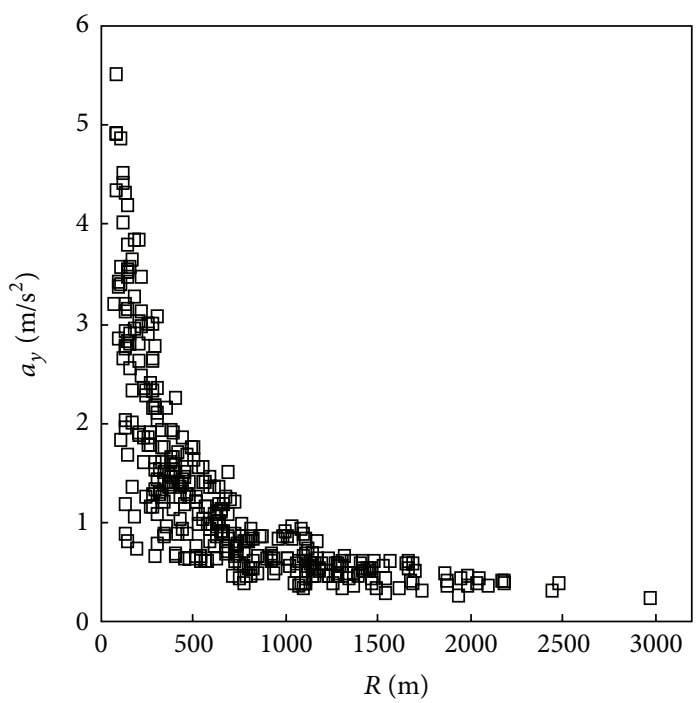

(f) Highway number 6 


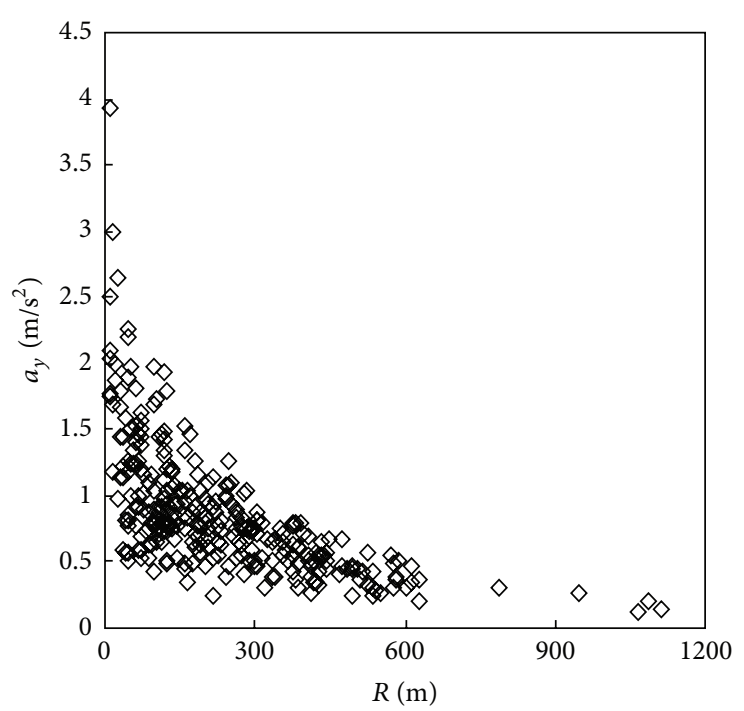

(g) Highway number 7

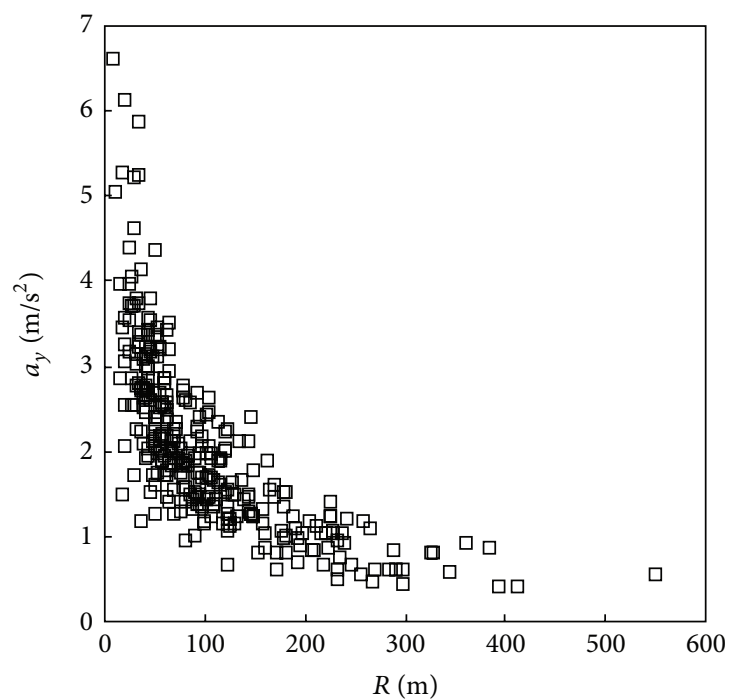

(i) Highway number 9

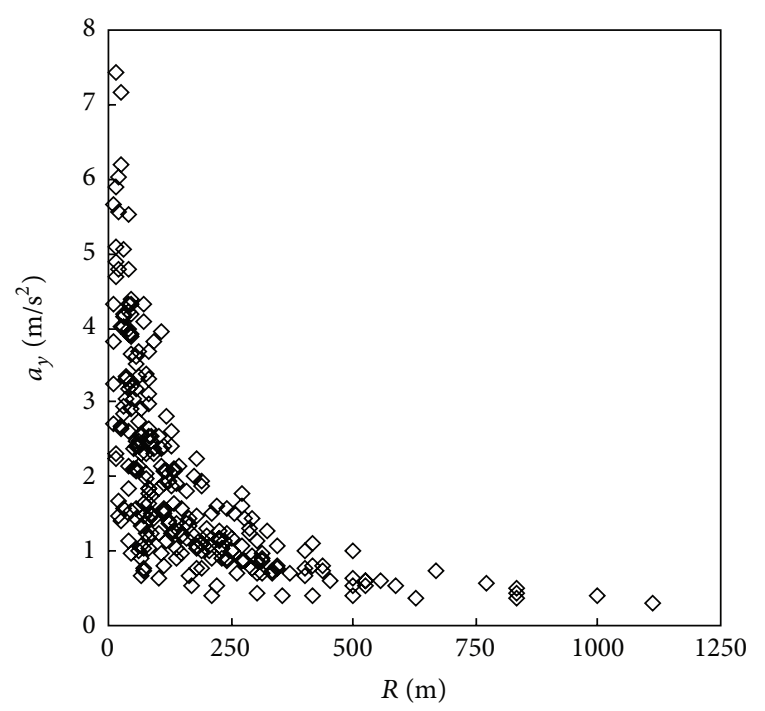

(k) Highway number 11

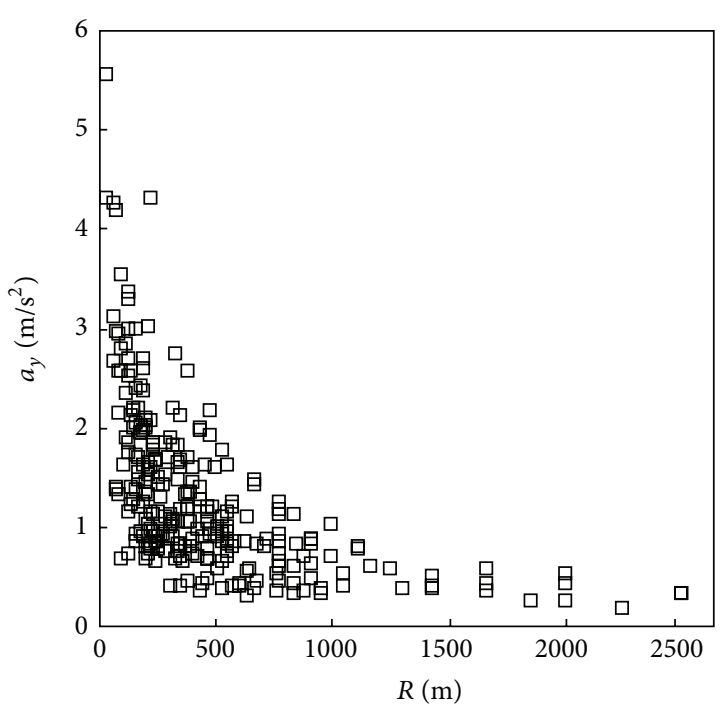

(h) Highway number 8

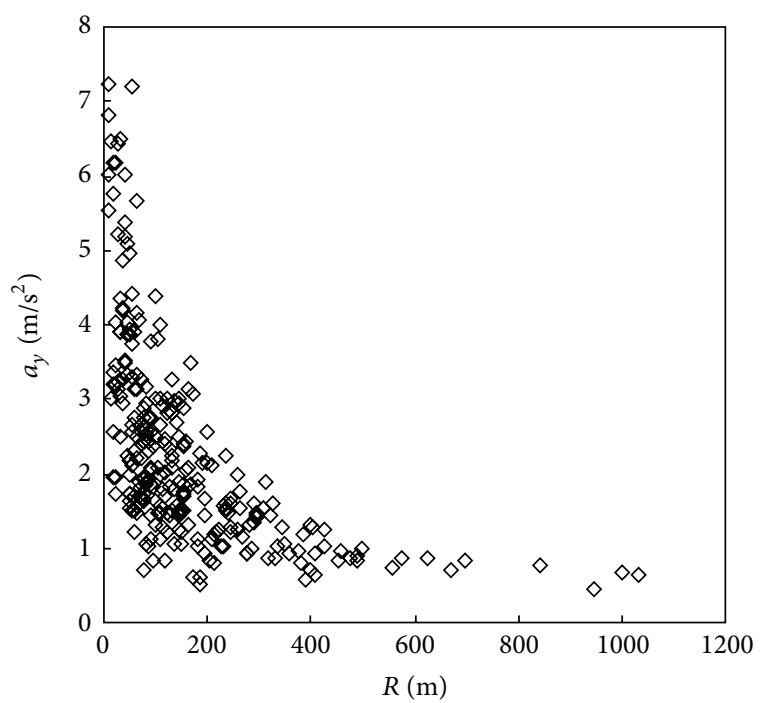

(j) Highway number 10

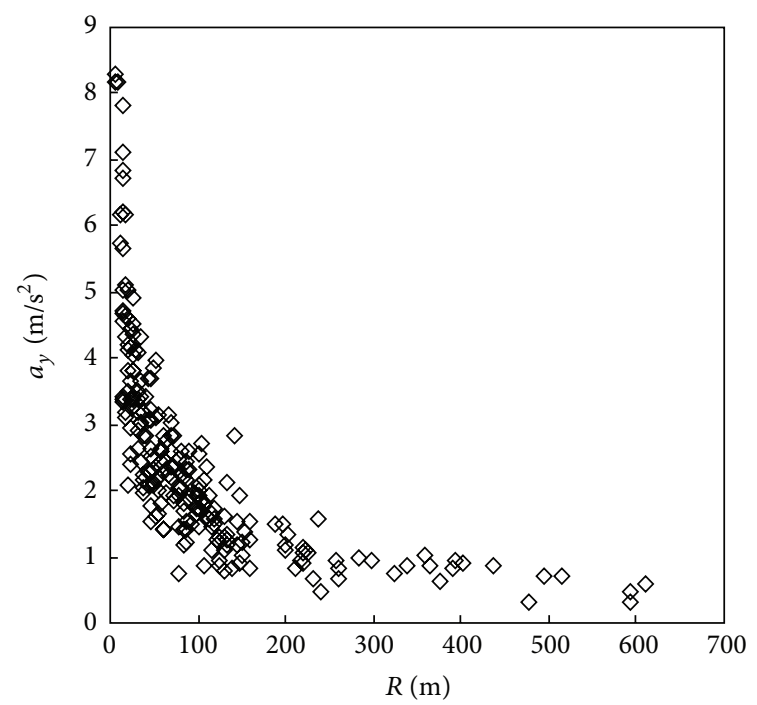

(l) Highway number 12

FiguRE 6: Scatter diagrams of lateral acceleration over radius. 


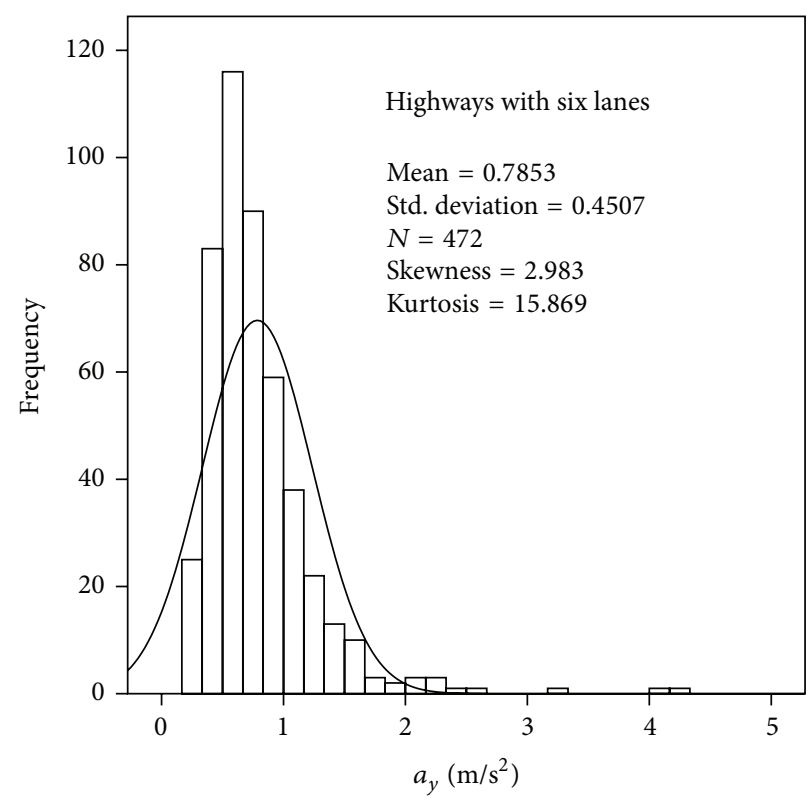

(a) Highway with six lanes

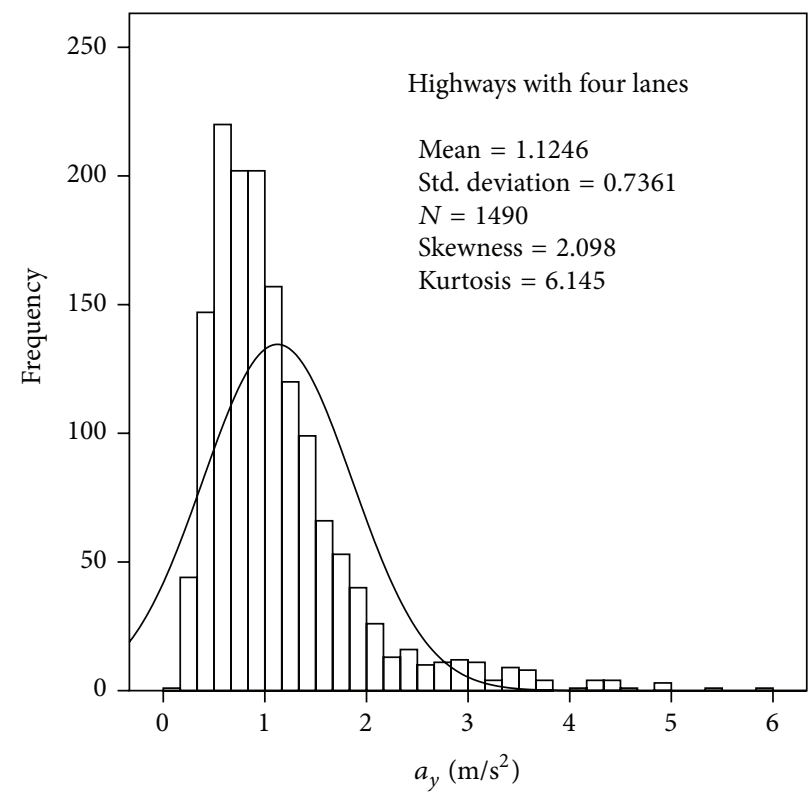

(b) Highway with four lanes

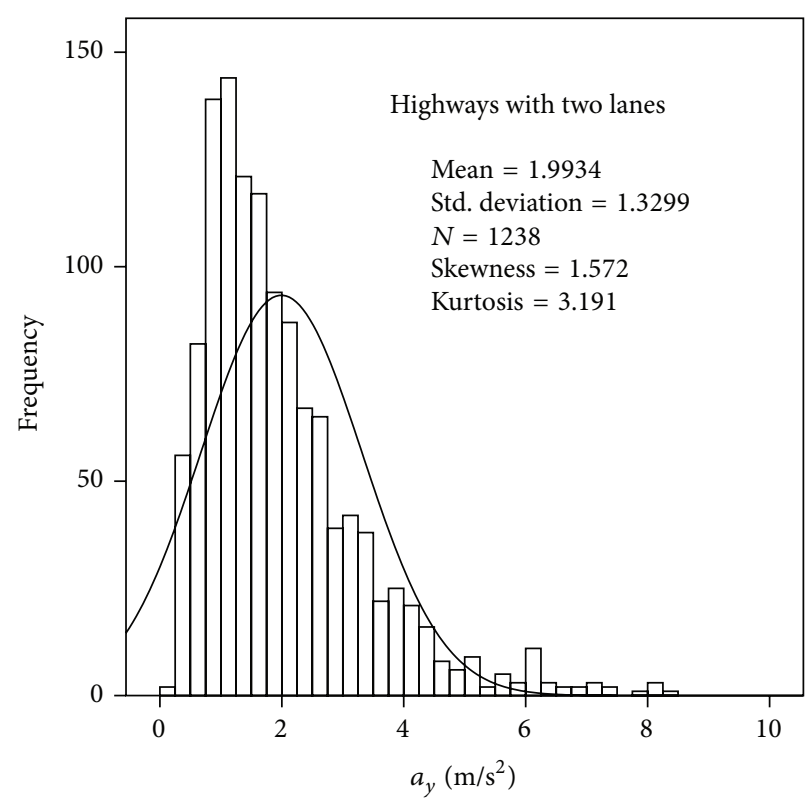

(c) Highway with two lanes

FIGURE 7: Normal distribution test results for lateral accelerations of three highway types.

The characteristic percentiles of the lateral acceleration were obtained in each speed section: the minimum value $\left(a_{y \text { min }}\right)$, maximum value $\left(a_{y \text { max }}\right), 50$ th percentile $\left(a_{y 50}\right), 85$ th percentile $\left(a_{y 85}\right)$, and average value $\left(a_{y \text { ave }}\right)$. As shown in Figure 10(b), the minimum value of lateral acceleration was almost constant, and the other lateral acceleration decreased with an increase in the speed. In addition, the average lateral acceleration was slightly greater than the 50th percentile value.
A unitary regression model was set up for each percentile line. The $a_{y 85}, a_{y 50}$, and $a_{y \text { ave }}$ values were slightly concave in form and negatively related to the speed. In addition, the average lateral acceleration $a_{y \text { ave }}$ and $a_{y 85}$ percentile were the most appropriate for the selection of geometric design parameters. Specifically, the average can be used for calculating the general values of geometric parameters, while the 85th percentile can be used for a limit value. The relations between $a_{y \text { ave }}, a_{y 85}$, and the speed can be expressed as follows: 

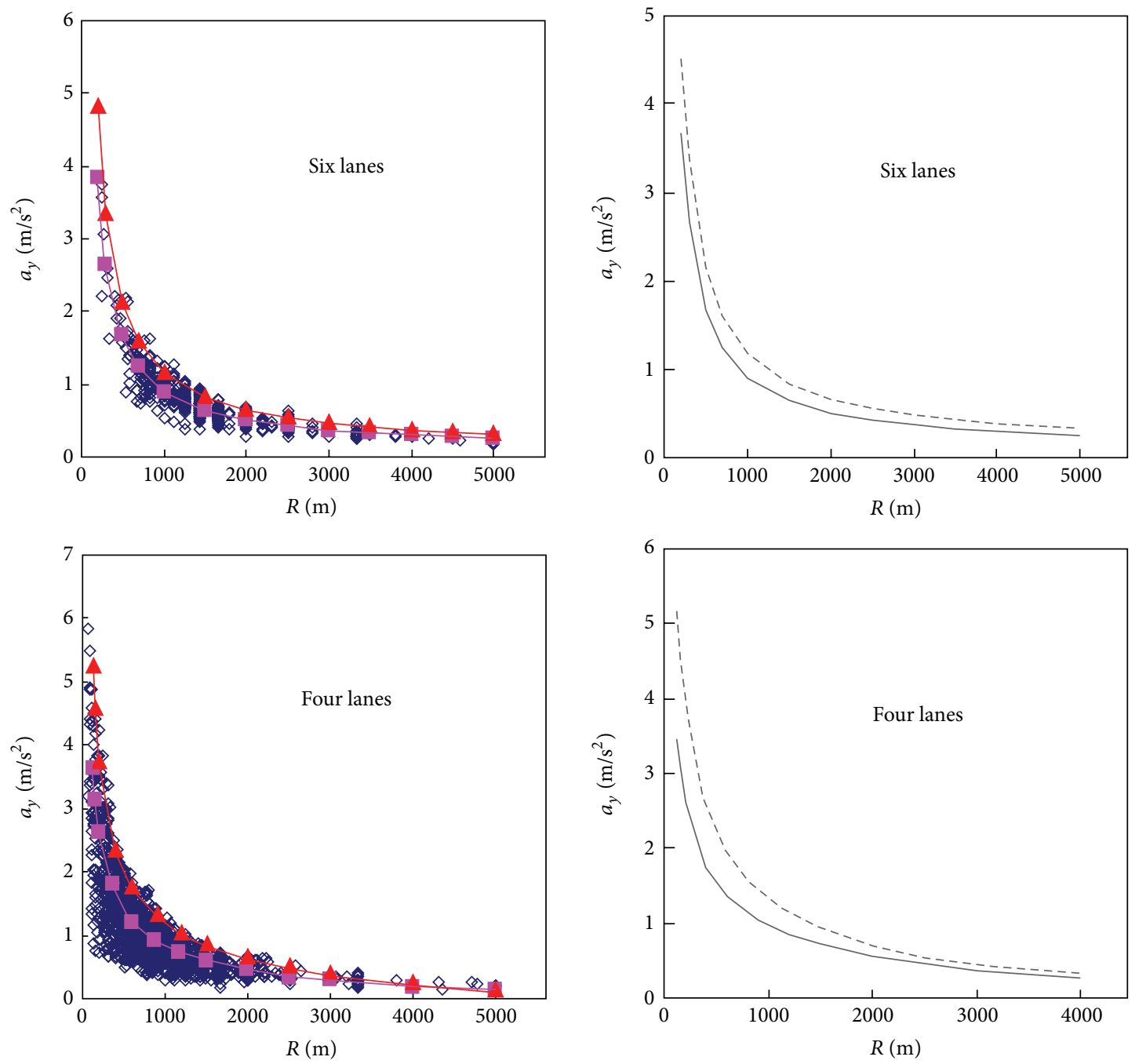

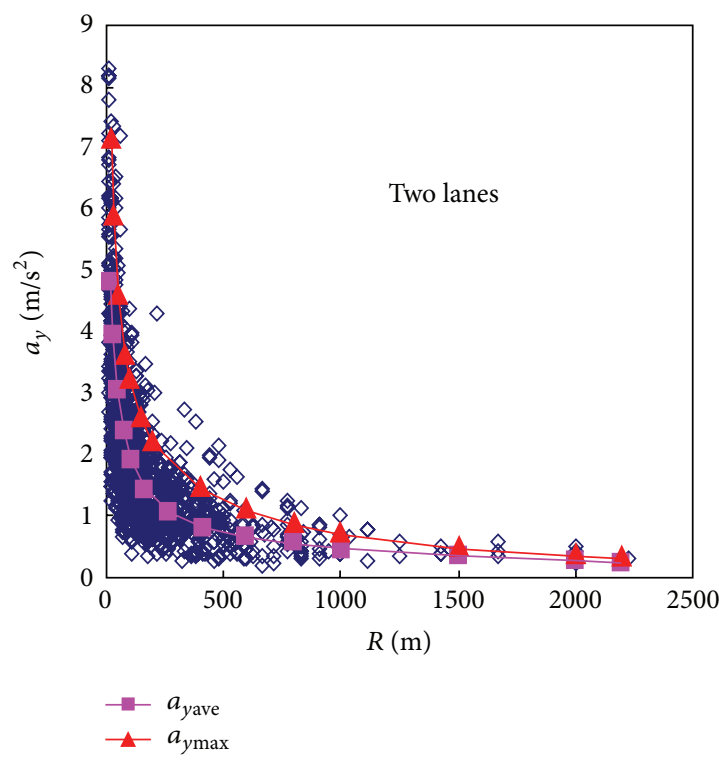

(a) Scatter diagram

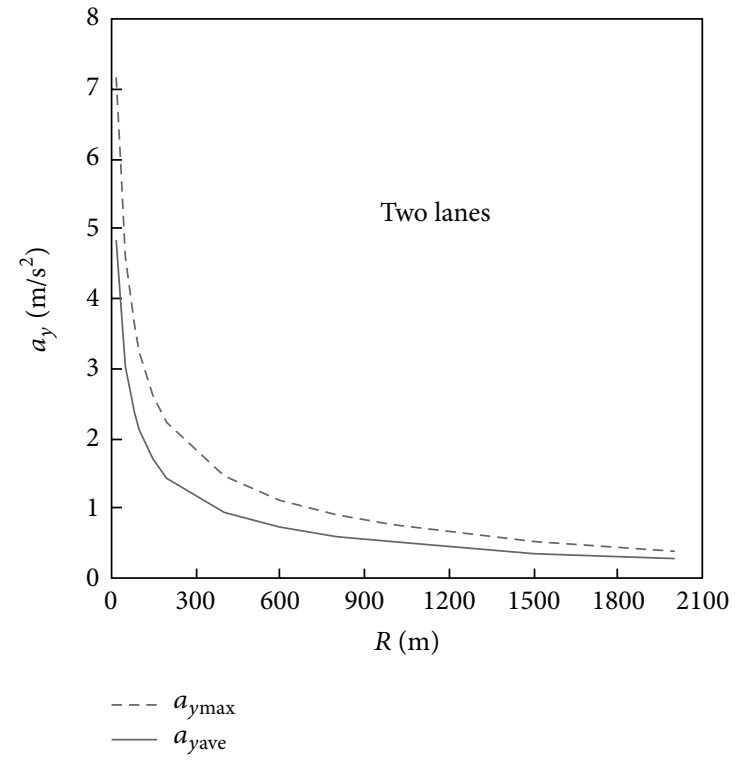

(b) Model curve of lateral acceleration over radius

FIGURE 8: Scatter diagram and model curve of lateral acceleration over radius, aggregated by type of passenger car. 


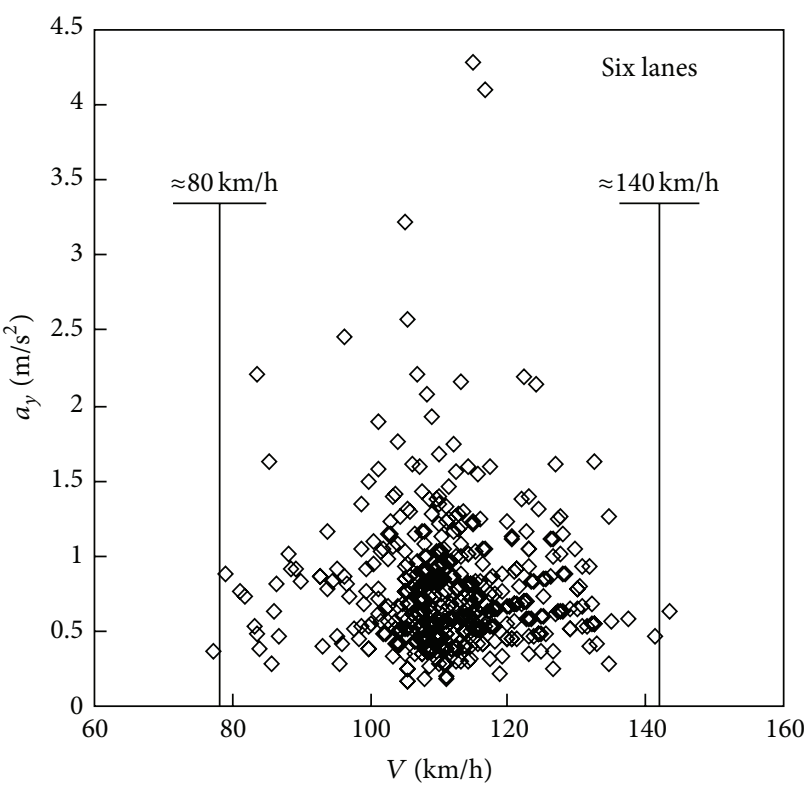

(a) Highway with six lanes

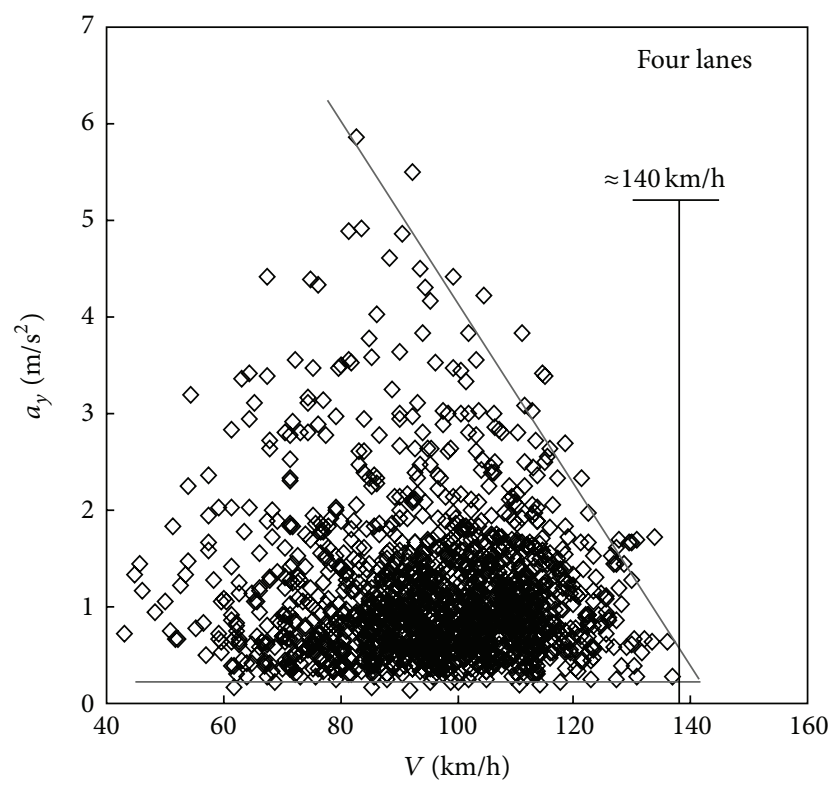

(b) Highway with four lanes

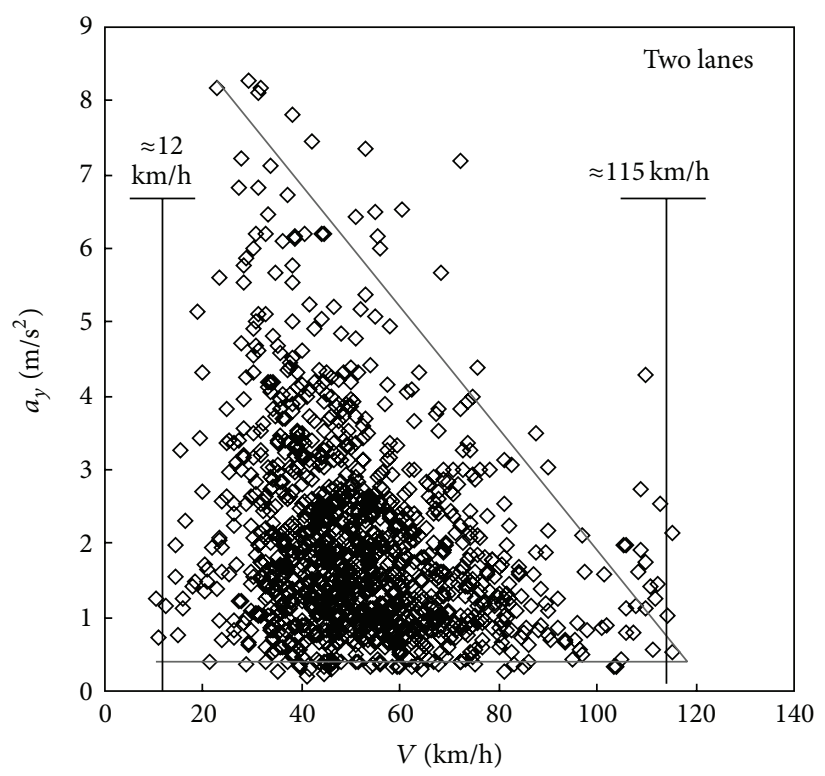

(c) Highway with two lanes

FIGURE 9: Scatter diagrams $\left(a_{y}-V\right)$ of three road types.

$$
\begin{array}{ll}
a_{y 85}=6.2217 e-0.0134 V & R^{2}=0.9732 \\
a_{y 50}=3.4984 e-0.0117 V & R^{2}=0.9761 .
\end{array}
$$

\section{Conclusions}

The driving characteristic parameters of a passenger car were measured and extracted from twelve highways with different design speeds and topographies. By synchronizing, matching, and counting the experimental data, the distribution of the lateral acceleration was obtained. The change laws for the lateral acceleration over the speed and curvature were analyzed. The main conclusions are as follows:
(1) The lateral accelerations of highways with six lanes were less than $3.5 \mathrm{~m} / \mathrm{s}^{2}$, and most were less than $1.8 \mathrm{~m} / \mathrm{s}^{2}$. It was comfortable to drive on a highway with six lanes. On the four-lane roads, the distribution of the lateral acceleration was almost the same as that on a highway with six lanes in a flat area. However, in a mountainous area, an increasing number of lateral accelerations exceeded the comfort limit, and a small part of the data exceeded the medium comfort limit $\left(3.6 \mathrm{~m} / \mathrm{s}^{2}\right)$. For highways with two lanes, the amplitude of the lateral acceleration was positively related to the terrain undulation and negatively related to 


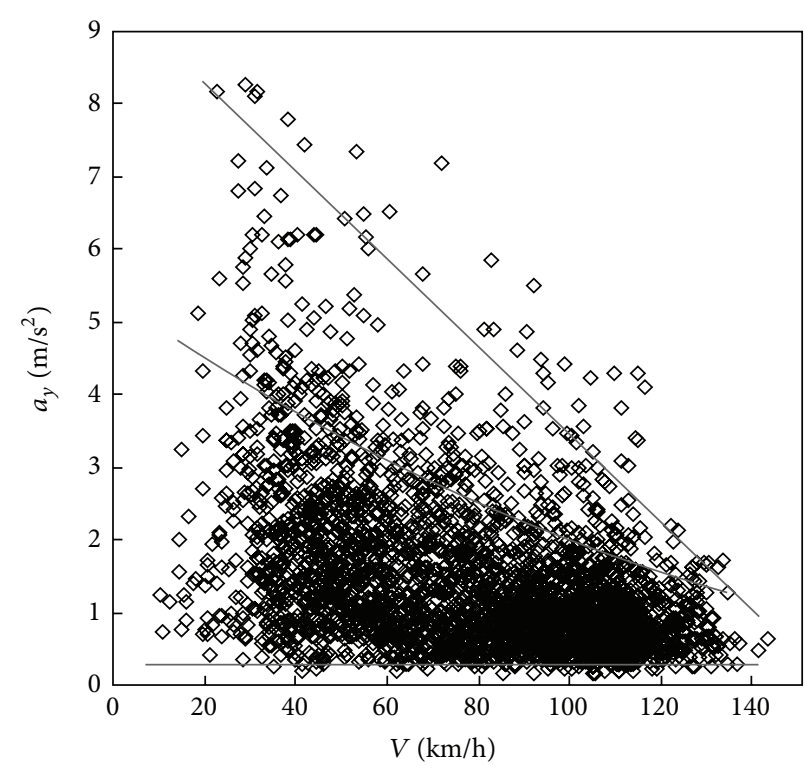

(a) The aggregated data

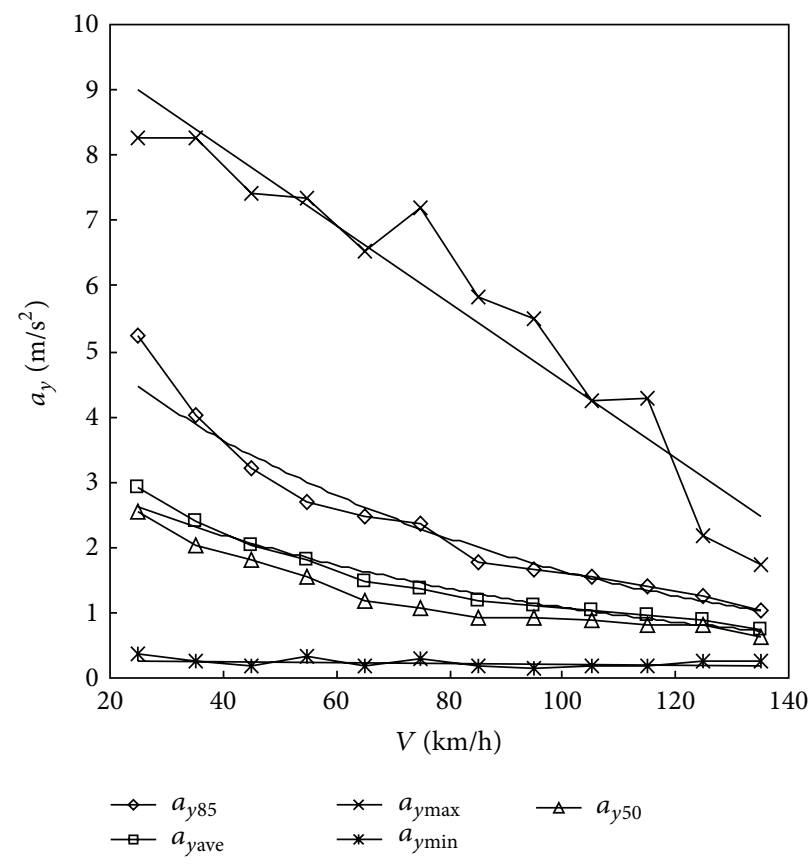

(b) Statistics regression analysis

FIGURE 10: Scatter diagram of lateral acceleration over speed, aggregated by three road types.

the design speed. With a design speed of $30 \mathrm{~km} / \mathrm{h}$ in a mountain area, a large proportion of the lateral accelerations exceeded the discomfort limit $\left(5 \mathrm{~m} / \mathrm{s}^{2}\right)$.

(2) According to the distribution characteristics of the lateral acceleration, the inflexion point appeared at approximately the 90th-92th percentiles, which was different from the 85th percentile found in the previous research. In addition, the maximum lateral acceleration exceeded the value of $8 \mathrm{~m} / \mathrm{s}^{2}$.

(3) The lateral acceleration was negatively related to the radius of the trajectory curvature; that is, the more moderate the latter, the smaller and more concentrated the former and vice versa. With regard to the road type, a greater number of lanes produced more concentrated data points.

(4) Models of the relationships between the lateral acceleration and the radius were set up for highways with six lanes, four lanes, and two lanes. For each type of vehicle, the average model and limit model were set up with different road types. The former described the driving behavior in a general case, which was dedicated to controlling the general values of geometric parameters; the latter represented the upper bound of the driving behavior, aiming at controlling the limit values of the geometric parameters.

(5) The lateral acceleration of a highway with six lanes was poorly related to the driving speed, which contrasted with highways with four lanes and two lanes. A stronger regularity for the data distribution turns was found for highways with four lanes and two lanes.
Based on the data gathered from all three road types, an aggregated scatter diagram showed that the lateral acceleration decreased as the curve radius increased or the speed increased.

(6) The characteristic percentiles of the lateral acceleration were analyzed statistically for several speed sections $(20-30,30-40, \ldots, 120-140)$, including the minimum $a_{y \min }$, maximum $a_{y \max }$, 50th percentile $a_{y 50}$, 85th percentile $a_{y 85}$, and arithmetic average $a_{y \text { ave }}$. The regression models of $a_{y \text { ave }}$ and $a_{y 85}$ over the speed were set up.

\section{Conflict of Interests}

The authors declare that there is no conflict of interests regarding the publication of this paper.

\section{Acknowledgments}

This research was supported by the National Natural Science Foundation of China (Grant no. 51278514) and Specialized Research Fund for the Doctoral Program of Higher Education (Grant no. 20135522110003).

\section{References}

[1] W. Hugemann and M. Nicke, "Longitudinal and lateral accelerations in normal day driving," in Proceedings of the Institute of Traffic Accident Investigators Conference (ITAI '03), E. J. Allen, Ed., pp. 1-8, Croydon, UK, 2003. 
[2] R. A. Tokunaga, M. Asano, K. Munehiro et al., "Effects of curve designs and road conditions on driver's curve sharpness judgment and driving behavior," Journal of the Eastern Asia Society for Transportation Studies, no. 6, pp. 3536-3550, 2005.

[3] P. J. Carlson and J. M. Mason Jr., "Relationships between ball bank indicator readings, lateral acceleration rates, and vehicular body-roll rates," Transportation Research Record, no. 1658, pp. 34-42, 1999.

[4] J. Bonneson, "Superelevation distribution methods and transition designs," NCHRP Report 439, Transportation Research Board, Washington, DC, USA, 2000.

[5] C. Tan, An investigation of comfortable lateral acceleration on horizontal curves [Ph.D. dissertation], Pennsylvania State University, State College, Pa, USA, 2003.

[6] S. He, C. L. Chang, and J. Z. Qian, "Study on correction of sideway force coefficient by measured speed," Journal of Highway and Transportation Research and Development, vol. 22, no. 6, pp. 54-56, 2005.

[7] X. D. Pan, T. Lin, and Z. Yang, "Relationship between the variation of drivers' heart rate and systolic blood pressure and the coefficient of transverse force of mountainous highways," Journal of Tongji University, vol. 34, no. 6, pp. 748-751, 2006.

[8] China Ministry of Communication, Technical Standard of Highway Engineering (JTG B01-2003), People’s Communication Press, Beijing, China, 2003.

[9] A. S. Kilinç and T. Baybura, "Determination of minimum horizontal curve radius used in the design of transportation structures, depending on the limit value of comfort criterion lateral jerk," 2012, http://www.fig.net/pub/fig2012/papers/ ts06g/TS06G_kilinc_baybura_5563.pdf.

[10] K. K. Dixon and J. Rohani, "Methodologies for estimating advisory curve speeds on oregon highways," Tech. Rep. FHWAOR-RD-08-04, FWHA, U.S. Department of Transportation, 2008.

[11] A. P. Voigt, D. W. Fenno, and D. W. Borchardt, "Evaluation of vehicle speeds on freeway-to-freeway connector ramps in Houston," Publication FHWA/TX-03/4318-1, Texas Transportation Institute, 2003.

[12] W. J. Cheng, Study on the evaluation method of highway alignment comfortableness [M.S. thesis], Hebei University of Technology, Tianjin, China, 2007. 


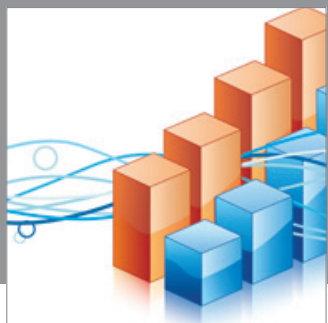

Advances in

Operations Research

mansans

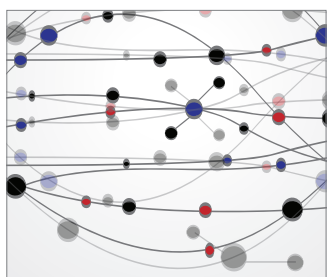

The Scientific World Journal
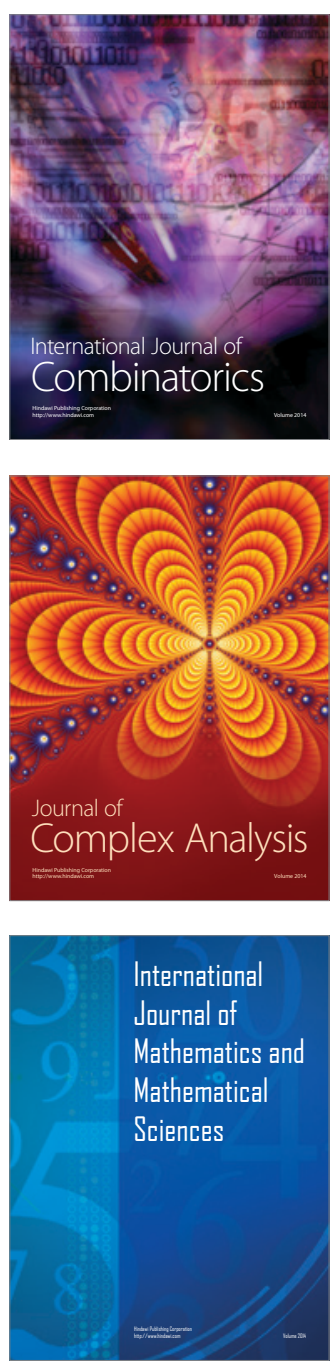
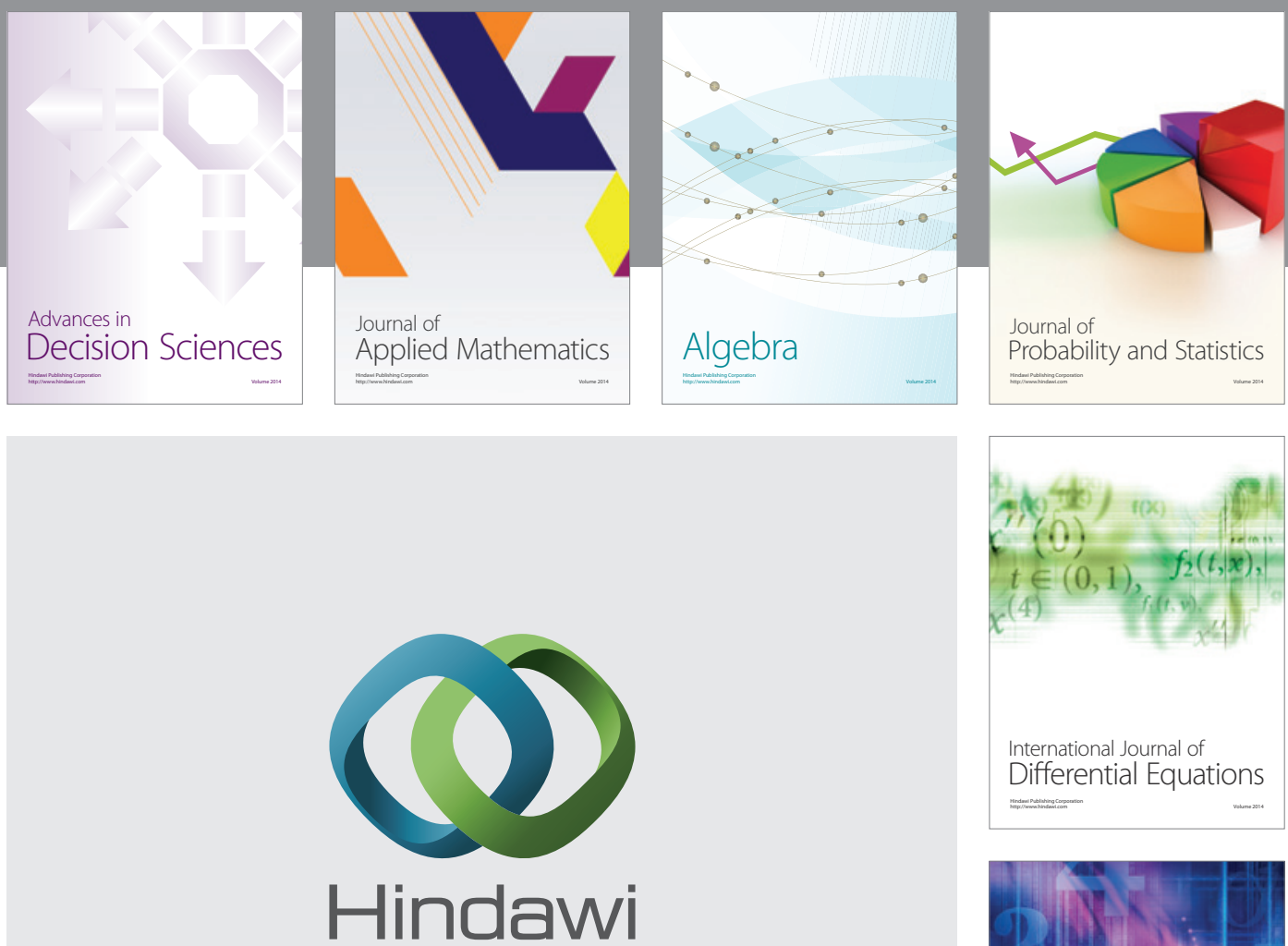

Submit your manuscripts at http://www.hindawi.com
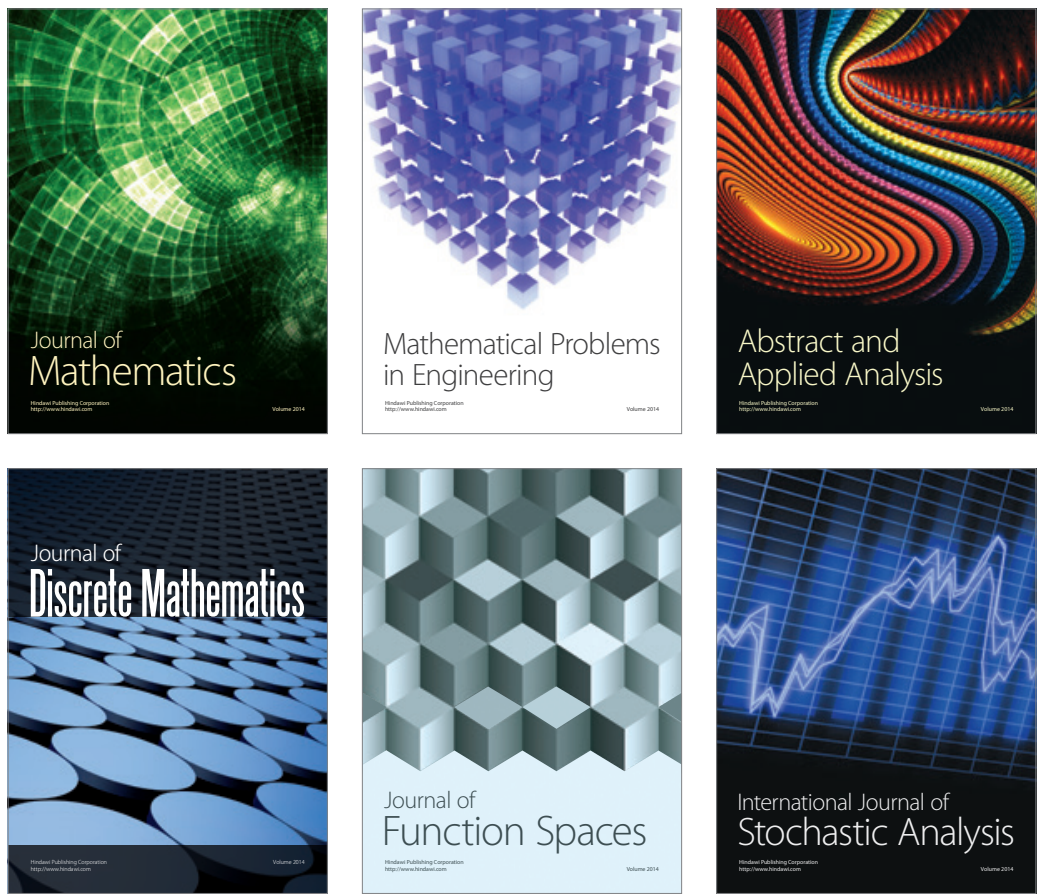

Journal of

Function Spaces

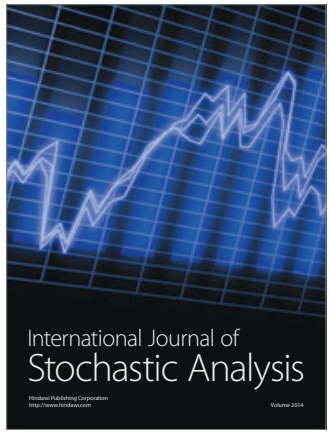

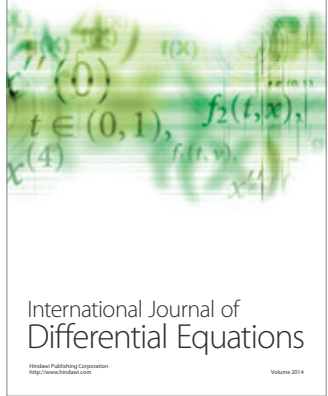
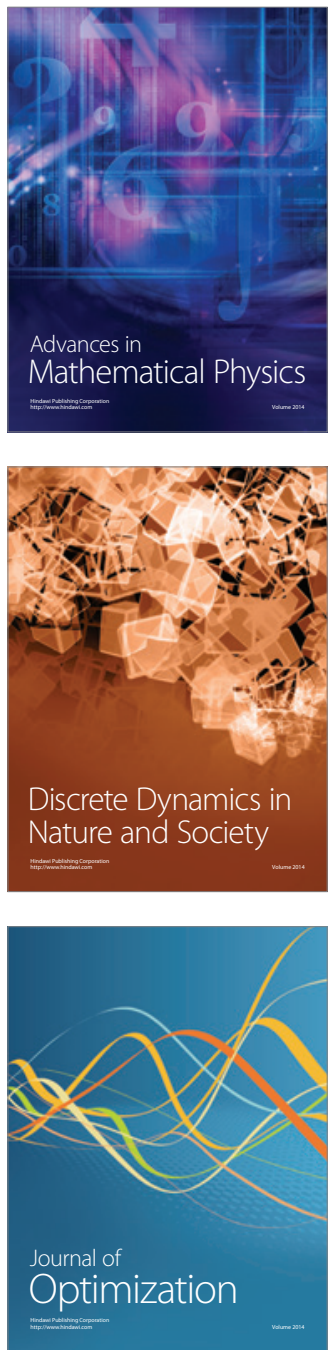